\title{
Design of single-phase chiral metamaterials for broadband double negativity via shape optimization
}

\author{
X. Y. $\operatorname{Lin}^{1}$, Eric Li ${ }^{2}$, Z. C. $\mathrm{He}^{1,3, *}$, Y. Wu ${ }^{1}$, Q. Q. $\mathrm{Li}^{1}$ \\ ${ }^{1}$ State Key Laboratory of Advanced Design and Manufacturing for Vehicle Body, Hunan University, \\ Changsha, 410082, P. R. China \\ ${ }^{2}$ School of Science, Engineering \& Design, Teesside University, Middlesbrough, UK \\ ${ }^{3}$ Guangxi Key Laboratory of Automobile Components and Vehicle technology, Guangxi University of \\ Science and Technology, Liuzhou, 545006, P. R. China
}

\begin{abstract}
Double negative elastic metamaterials are generally obtained by inducing the combination of multiple resonance with different negative parameters. However, these approaches yield relatively narrow frequency ranges and require multiple-phase materials, limiting their practical applications. This paper demonstrates a strategy to design a type of single-phase elastic chiral metamaterials exhibiting broadband double negativity (negative mass density and negative bulk modulus). The mechanism of broadband double negative property is firstly studied. Subsequently, the design approach to optimize the frequency range of double negativity is constructed via the shape optimization formulation. Three numerical examples are presented to validate the proposed method. Broadband double negativity is then demonstrated through the simulation of the negative refraction and the imaging at a sub-wavelength scale. Interestingly, the mode conversion has been observed at the interface, and a super-resolution image of $0.28 \lambda$ that breaks through the Rayleigh diffraction limit is achieved. The proposed metamaterial combined with the design approach is a very efficient way to obtain double negativity over a broad frequency band and it may thus have great potential for designing new elastic metamaterials.
\end{abstract}

Key words: Elastic metamaterials; Broadband double negativity; Negative refraction; Super-resolution imaging.

\footnotetext{
${ }^{1}$ Corresponding author

Email address: hezhicheng815@hnu.edu.cn (Z. C. He)
} 


\section{Introduction}

Double negative electromagnetic metamaterials are a kind of artificial materials with engineered microstructures that can exhibit negative dielectric constant and magnetic permeability in a specific frequency range [1-3]. Owing to the unique double negative properties, rich physical phenomena such as the negative refraction [1], a perfect lens [2], and super-resolution imaging [4] can be observed. Based on the double negative concept, many efforts have been devoted to the realization of acoustic/elastic double negativity [5-10]. In the acoustic/elastic metamaterials (AMMs or EMMs), the propagating waves are mainly longitudinal and transverse waves. Their propagating properties can be characterized by three independent material constants which are the bulk modulus, the shear modulus, and the mass density. However, none of them presents negative property in the conventional materials $[5,11$, 12].

The research on the novel acoustic/elastic metamaterials has shown that the negative shear modulus is resulted from the quadrupolar resonance [13]. The negative bulk modulus is generated by inducing the monopolar resonance with which a structure can produce volume expansion (compression) under the compressive (expansive) excitation [14]. Besides, the negative mass density emerges when the host medium and the resonator's mass display relative motion with an opposite phase as a result of the dipolar resonance [15]. Therefore, the double negative property in acoustic/elastic fields can be realized by combining two different counterparts of different resonance mechanisms. For example, a metamaterial composed of side hole unit with the monopolar resonance and a thin membrane element with dipolar resonance is able to present negative bulk modulus and negative mass density simultaneously [16]. Unfortunately, there are many limitations of these approaches when adopting the combination of multiple materials. Because the frequency range of double negative parameters of current metamaterials is very narrow, and the materials mixing scheme requires complex adjustments of the geometry parameters of 
multiple-phase materials, which will bring many difficulties in the manufacturing [7]. Consequently, solving these two issues is of great significance [17]. To conquer these limitations, many efforts have been made.

In the aspect of systematic design strategy, the topology optimization-based method is employed to realize a desired negative refraction in a metamaterial slab at a predetermined angle of incidence [18]. In a similar way, the bi-directional evolutionary structural optimization is proposed to search the best topology of a structure with broadband all-angle negative refraction [19]. Inspired by the principle of the topology optimization, Dong et al. $[8,10]$ developed a genetic-algorithm-based approach combined with the effective medium approach for the design of two-dimensional single-phase acoustic/elastic metamaterials. In their strategy, the frequency range of double negativity is recognized as an objective index and required to be maximized. These recent discoveries have shown that a design strategy with a systematic optimization approach is a very effective way to obtain the desired elastic metamaterials with double negative properties.

Another aspect of enlarging the frequency range of double negativity is the development of resonance mechanism of the elastic metamaterials. By introducing the electrical components, the active and adaptive acoustic/elastic metamaterials have been used to provide a controllable or tunable property [20-22]. In addition to the electro-mechanical coupling mechanism, the elastic metamaterials with chiral effect have also gain a lot of research interest $[23,24]$. To be more specific, the behavior of chirality is that a pure rotation can generate a shrinking or a dilatation in a chiral metamaterial with rotational symmetry topology. As this type of structures are non-superimposable on its mirror image [25], the intriguing phenomenon cannot be observed in those structures with mirror/orthogonal symmetry. In this situation, the additional material parameters (e.g., chirality index) of the ortho-chiral EMMs only reverse its sign and maintain the invariance of the strain energy density when the handedness of the material pattern is flipped over $[24,26]$. By virtue of the chiral effect, Liu et al. [27] engineered a chiral elastic metacomposite composed of a 
hexagonal lattice and a inclusion, with which the negative bulk modulus and the negative density can be obtained simultaneously. Based on the concept of chirality, the chiral metacomposite is further developed as a two-dimensional chiral continuum, which is composed of the frame, the connecting beams, and the lumped mass [28]. Such a type of chiral metamaterial shares some common properties with the lattice structure. The first common property is that the mechanical behavior of the chiral lattice structure is significantly affected by the different geometrical configurations [23]. The second one is that the double negativity of the chiral metamaterials is also influenced by the geometry parameters. Hence, it is promising to enlarge the frequency range of the double negativity by carefully selecting the parameters of the single-phase chiral metamaterials.

In light of this, a novel strategy to the problem of designing elastic metamaterials exhibiting a broadband negative mass density and negative bulk modulus is studied. The strategy is based on employing a shape optimization to maximize the frequency bandwidth of the double negativity of the single-phase chiral metamaterial in the prescribed target frequency range, which has not yet been studied. The remainder of this paper is structured as follows. Section 2 describes the model of single-phase chiral elastic metamaterials (EMMs) and the effective medium approach, where the latter is used to capture the effective parameters. The double negative mechanism is also addressed in this section. Section 3 presents the influences of the geometry parameters on the bandwidth of double negativity. And the optimization design strategy is proposed. Then, several numerical examples are carried out to demonstrate the effectiveness of the proposed optimization strategy. In Section 5, the related phenomena including the negative refraction and the imaging are depicted to realize the double negative properties of single-phase chiral metamaterial. Finally, some conclusions are summarized in Section 6.

\section{Foundation of single-phase chiral elastic metamaterials}

Figure 1 shows the schematic of the proposed single-phase chiral metamaterial. The initial chiral unit cell with square lattice contains three components including the 
outer frame, the four connecting beams and the inner core. In the figure, the white part between the frame and the inner core is the structure gap. The geometry and the material property parameters are listed in Tab. 1.
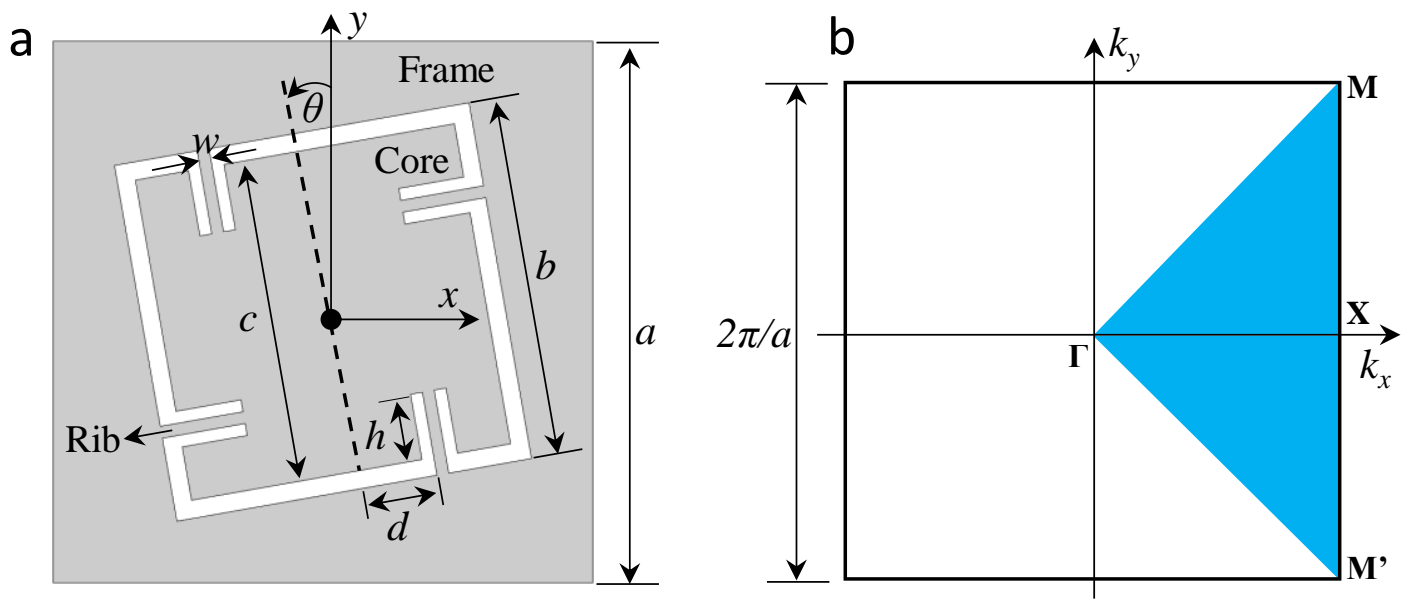

Fig. 1 (a) The geometry of the single-phase chiral EMM, and (b) the first Brillion zone.

Tab. 1 Geometry and material parameters of the chiral EMM

\begin{tabular}{cccc}
\hline Geometry parameters $(\mathrm{mm})$ & \multicolumn{2}{c}{ Material property parameters } \\
\hline$a$ & 45 & Young's modulus & $200 \mathrm{GPa}$ \\
$b$ & 30 & Mass density $\rho$ & $7850 \mathrm{~kg} / \mathrm{m}^{3}$ \\
$c$ & 27 & Poisson's ratio & \\
$d$ & 8 & \\
$h$ & 11 & \\
$\theta($ degree $)$ & 10 & \\
$w$ & 1 & \\
\hline
\end{tabular}

Neglecting the body forces, the governing equation for an linear elastic solid is expressed as [8]:

$$
(\lambda+2 \mu) \nabla[\nabla \cdot \mathbf{u}(\mathbf{r})]-\mu \nabla \times[\nabla \times \mathbf{u}(\mathbf{r})]+\rho \omega^{2} \mathbf{u}(\mathbf{r})=0
$$

where $\lambda$ and $\mu$ are the Lamé's constants. $\nabla=(\partial / \partial x, \partial / \partial y)$ denotes the gradient operator, and $\mathbf{u}(\mathbf{r})=\left(u_{x}, u_{y}\right)$ represents the displacement at the position $\mathbf{r}=(x, y) . \omega$ is the circular frequency of the propagating wave.

The constitutive relationship in the 2-D solid is expressed as $\boldsymbol{\sigma}=\mathbf{D} \boldsymbol{\varepsilon}$, where $\boldsymbol{\sigma}$ represents the stress vector, $\boldsymbol{\varepsilon}$ denotes the strain vector, and $\mathbf{D}$ is the symmetric positive definite matrix of material. They can be written as follows: 


$$
\boldsymbol{\sigma}=\left[\begin{array}{c}
\sigma_{x x} \\
\sigma_{y y} \\
\tau_{x y}
\end{array}\right], \quad \mathbf{D}=\left[\begin{array}{ccc}
\lambda+2 \mu & \lambda & 0 \\
\lambda & \lambda+2 \mu & 0 \\
0 & 0 & \mu
\end{array}\right], \boldsymbol{\varepsilon}=\left[\begin{array}{c}
\varepsilon_{x x} \\
\varepsilon_{y y} \\
\gamma_{x y}
\end{array}\right]
$$

where $\varepsilon_{x x}=\partial u_{x} / \partial x, \varepsilon_{y y}=\partial u_{y} / \partial y, \gamma_{x y}=1 / 2\left(\partial u_{y} / \partial x+\partial u_{x} / \partial y\right)$.

Using Eq. (2) and rewriting Eq. (1) in $x$ and $y$ directions respectively yield:

$$
\begin{aligned}
& (\lambda+2 \mu) \frac{\partial^{2} u_{x}}{\partial x^{2}}+(\lambda+\mu) \frac{\partial^{2} u_{y}}{\partial x \partial y}+\mu \frac{\partial^{2} u_{x}}{\partial y^{2}}+\rho \omega^{2} u_{x}=0 \\
& \mu \frac{\partial^{2} u_{y}}{\partial x^{2}}+(\lambda+\mu) \frac{\partial^{2} u_{x}}{\partial x \partial y}+(\lambda+2 \mu) \frac{\partial^{2} u_{y}}{\partial y^{2}}+\rho \omega^{2} u_{y}=0
\end{aligned}
$$

Based on the Bloch's principle [29], the displacement vector in a periodic solid system can be expressed as $\mathbf{u}(\mathbf{r})=e^{i(\mathbf{k} \cdot \mathbf{r})} \mathbf{u}_{\mathbf{k}}(\mathbf{r})$, in which the displacement vector $\mathbf{u}_{\mathbf{k}}(\mathbf{r})$ and the spatial position vector $\mathbf{r}$ share the same periodicity. $\mathbf{k}=\left(k_{x}, k_{y}\right)$ is the Bloch wave vector. Based on the standard Galerkin approach, Eqs. (3)-(4) can be rewritten in a matrix form [30]:

$$
\left[\mathbf{K}-\omega^{2} \mathbf{M}\right] \mathbf{U}=0
$$

where $\mathbf{U}$ represents the displacements, $\mathbf{K}$ denotes the stiffness matrix, and $\mathbf{M}$ is the mass matrix. To solve the dispersion problem described in Eq. (5), the periodic Bloch-Floquet boundary condition should be introduced. The points of wave vector are restricted to the boundaries of blue irreducible Brillion zone, as expressed in Fig. 1(b) [31].

\subsection{Effective medium approach}

In this subsection, the homogenization approach used to extract the effective dynamic properties of the chiral EMMs is introduced. It is noted that the elastic metamaterial can be considered as a homogeneous medium and acts as a unified system under the long-wavelength assumption [14]. In the present work, the long-wavelength assumption admits that $\lambda \geq 6.5 a$ (where $\lambda$ is the wavelength of the 
incident wave and $a$ is the lattice constant). By using the effective medium approach, the effective parameters are hence calculated through the boundary-average technique. Specifically, the corresponding eigenstates are applied on the boundaries, whereas, the dynamic responses including the effective forces, macro-strains, displacements and accelerations of the unit cell are calculated accordingly.

To determine the effective mass density, the boundaries of the unit cell expressed in Fig. 1(a) are subjected to an additional excitation displacement field $\mathbf{u}^{b}=\hat{\mathbf{u}}^{b} e^{i \omega t}$, where $\hat{\mathbf{u}}^{b}$ is the prescribed amplitude. Thus, the effective mass density is computed by using the Newton's second law [32]:

$$
\rho_{\text {eff }}(\omega)=\frac{m_{\text {eff }}}{V}=-\frac{\left|\mathbf{F}_{\text {eff }}\right|}{V\left|\ddot{\mathbf{u}}^{b}\right|}=-\frac{\left|\mathbf{F}_{e f f}\right|}{V \omega^{2}\left|\hat{\mathbf{u}}^{b}\right|}
$$

where $m_{\text {eff }}$ represents the effective mass, and $\mathbf{F}_{\text {eff }}$ denotes the averaged effective forces exerted on the boundary. In the $2 \mathrm{D}$ case, $V$ denotes the area of the unit-cell. The details of the computation of the averaged effective force can be found in Ref. [14, 27].

Another aspect of the effective dynamic parameters is the determination of the effective moduli. Generally, the macro-stress field $\boldsymbol{\sigma}^{b}$ of a unit cell is defined through the averaging of micro-stress $\boldsymbol{\sigma}$. The macro-strain field $\mathbf{E}^{b}$ is computed by the averaging of micro-strain $\varepsilon$ in the external boundaries of the representative volume element (RVE), i.e., $\partial V$. This can be written as:

$$
\mathbf{E}^{b}=\frac{1}{V} \int_{\partial V} \boldsymbol{\varepsilon} \mathrm{d} s, \boldsymbol{\sigma}^{b}=\frac{1}{V} \int_{\partial V} \boldsymbol{\sigma} \mathrm{d} s
$$

where the integral is applied along the boundary.

To estimate the effective bulk modulus and shear modulus, the corresponding eigenstates including the global hydrostatic strain filed $\mathbf{E}_{K}^{b}$ and the global shear strain filed $\mathbf{E}_{S}^{b}$ can be expressed as [32]:

$$
\mathbf{E}_{K}^{b}=\varepsilon_{K}\left[\begin{array}{ll}
1 & 0 \\
0 & 1
\end{array}\right] \quad \mathbf{E}_{S}^{b}=\varepsilon_{S}\left[\begin{array}{cc}
1 & 0 \\
0 & -1
\end{array}\right]
$$

where $\varepsilon_{K}$ and $\varepsilon_{S}$ denote the amplitudes of the imposed strain excitations. By using 
the relationship between the global displacement field and the imposed strain excitations, the global displacements applied on the boundaries are determined by [27]:

$$
\tilde{\mathbf{u}}^{b}(\mathbf{x})=\mathbf{E}^{b} \cdot \mathbf{x}
$$

where $\mathbf{x}=\mathbf{r}$ is the position vector, and $\tilde{\mathbf{u}}^{b}(\mathbf{x})$ is the macro-strain. In order to determine the effective moduli, the equivalence between the effective model and the homogenous model is required. This suggests that the work of external force on the effective model should be equivalent to the strain energy of the homogenous continuum. Thus, the effective moduli can be computed by [27]:

$$
\begin{gathered}
K_{e f f}(\omega)=\frac{W_{K}}{4 \varepsilon_{K}^{2} V}=\frac{1}{4 \varepsilon_{K}^{2} V} \cdot\left(\sum_{i=1}^{4} \mathbf{F}_{i}^{b} \cdot \mathbf{u}_{i}^{b}\right) \\
G_{e f f}(\omega)=\frac{W_{G}}{4 \varepsilon_{S}^{2} V}=\frac{1}{4 \varepsilon_{S}^{2} V}\left(\sum_{i=1}^{4} \mathbf{F}_{i}^{b} \cdot \mathbf{u}_{i}^{b}\right)
\end{gathered}
$$

where $\mathbf{F}_{i}^{b}$ is the resultant force on the $i$-th boundary, which can be computed through the similar way as the effective forces. $K_{\text {eff }}$ is the effective bulk modulus and $G_{\text {eff }}$ is the effective shear modulus. $W_{K}$ and $W_{G}$ denote the work of the deformed representative volume element when applying the global hydrostatic strain filed and the shear strain filed, respectively. In addition, the effective elastic modulus along the $\Gamma \mathrm{X}$ direction (see Fig. 1(b)) is defined as $E_{\text {eff }}=K_{\text {eff }}+G_{\text {eff. }}$ With the effective mass density $\rho_{\text {eff, }}$, the velocity of the longitudinal wave can be computed as $v_{l}=\sqrt{E_{\text {eff }} / \rho_{\text {eff }}}$.

Remark 1. Since the boundary-average technique is applied to extract the effective parameters, it is noted that the number of the degree of freedoms at the boundaries is of great importance [24]. Generally, an exact result of the resultant forces or the displacements can be obtained by enriching the degree of freedoms for the boundaries of the RVE using the finite element method [33]. In this case, it is suggested that an appropriate refinement is required during the computation of the effective parameters.

\subsection{Modal analysis and double negative mechanism}

In this subsection, the mechanism of the double-negativity will be discussed by 
using the dispersion curve and the effective parameters of the chiral unit cell. As shown in Fig. 2(a), the band structures of the microstructure with the parameter of $b=30, c=27$, $d=8, h=11, \theta=0$, and $w=1$ are plotted. The vector $\mathbf{X}$ is defined as $\mathbf{X}=[b, c, d, h, \theta, w]^{\mathrm{T}}$ which is a $6 \times 1$ dimensional vector that contains the six geometry parameters (the lattice constant $a$ is fixed and is not involved here). To clearly track the wave modes from the second to the fourth bands, six wave modes corresponding to the $k$-points are depicted in Fig. 2. The considered chiral EMM in the present work is a $\mathrm{Z}_{4}$-invariance structure which has 4-fold of rotational symmetry. Therefore, the dispersion curves along ГМ and $\Gamma M^{\prime}$ ' (see Fig. 1(b)) are symmetrical with respect to the $\mathrm{X}$ point $[27,34]$, these two directions will be treated as the same in the following.

In the second band, the mode shape (S2) of $\Gamma$ point denotes the rigid motion of the chiral EMM; while the mode shapes of S1 and S3 indicate the mixed translational motion of the core and these mode shapes are very similar to those of S4. In the third band, the mode shapes of X point (S4) show the translational motion of the core, but the mode shapes near $\Gamma$ (along $\Gamma \mathrm{X}$ direction) display the rotational deformation of the core. Along the $Г \mathrm{M}$ direction, the mode shapes of S5 and S6 which are the core's rotational resonance dominate the deformation of the whole structure, and the frame is almost static. In the fourth band, the mode shapes S7-S8 indicate that the main deformation of the EMMs is the relative motion between the frame and the core along the $Г \mathrm{M}$ direction. And the motions of the four sides of the frame represent the main deformation of the microstructure. Overall, the second and fourth bands represent the translational oscillation of the core along the $\Gamma \mathrm{X}$ and the $\Gamma \mathrm{M}$ directions, respectively, and the vibration pattern of the third band are mainly the rotational oscillation. These two different oscillation modes are contributed to the generation of the negative mass density and the negative bulk modulus.

Another notable feature of Fig. 2 is that the slopes of the third band curve along the $\Gamma X$ path are negative. These $k$-points with the negative curve slope form a frequency range of $[16.2 \mathrm{kHz}-18.4 \mathrm{kHz}]$, which is a frequency range with negative 

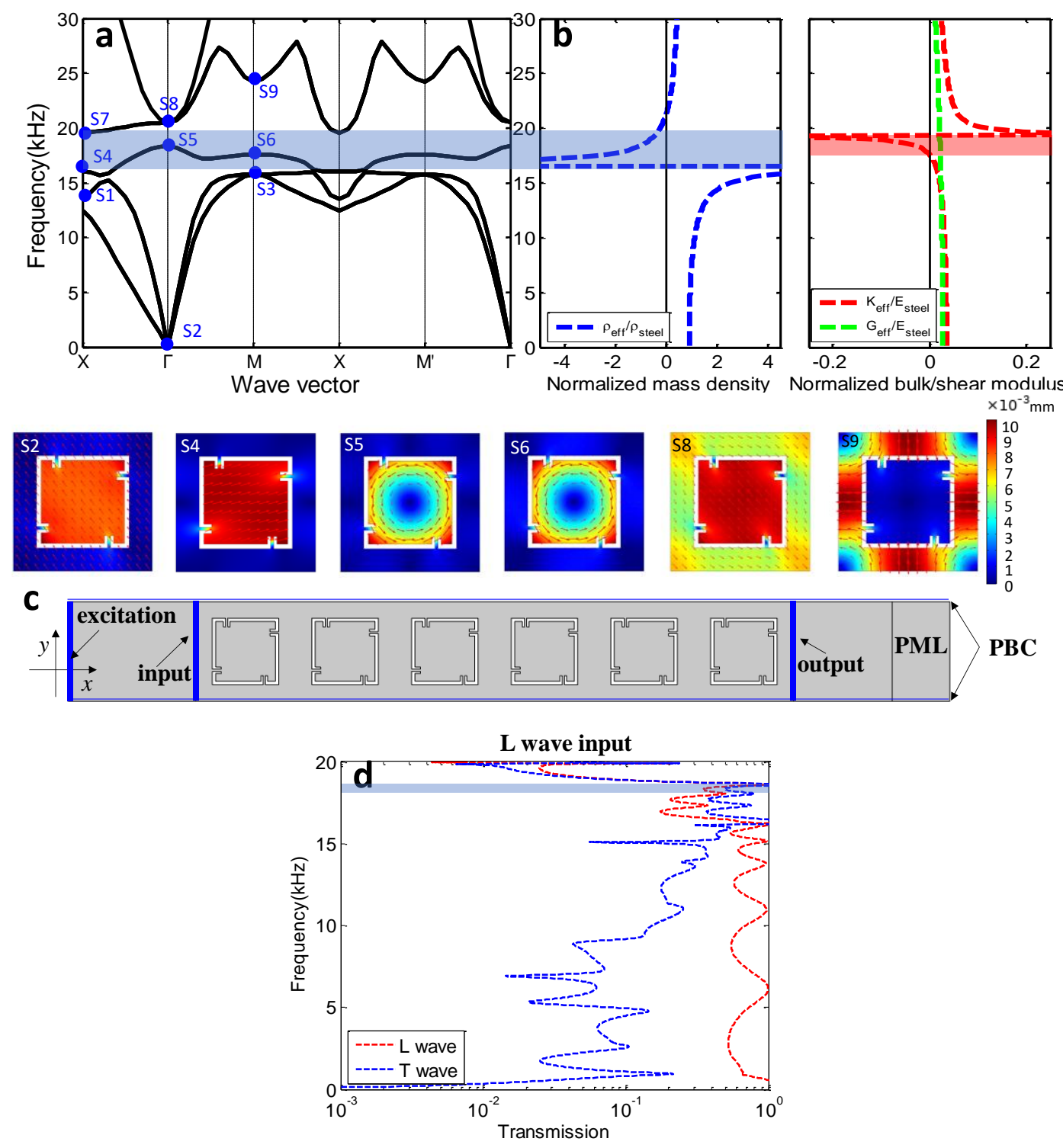

Fig. 2 Band structures and modes of the single-phase EMM. (a) The band structure of the chiral EMMs; (S1-S9) the modes corresponding to the blue $k$-points denoted in 2nd-4th bands. Note that the red dotted arrow represents the particle displacement, and its length indicates the magnitude of the displacement. (b) The negative properties curve. (c)The numerical setup for the computation of the transmission. (d-e) The transmission along $\Gamma X$ direction when (d) the longitudinal wave is impinging to the sample.

group velocity. Meanwhile, the stopband between the third and the fourth bands curve is $[18.4 \mathrm{kHz}-19.6 \mathrm{kHz}]$, which is generated due to the local resonance. Besides, there is a frequency range $[16.5 \mathrm{kHz}-19.9 \mathrm{kHz}]$ showing the negative mass density and a range [17.6kHz-18.4kHz] exhibiting the negative bulk modulus, as show in Fig. 2(b). The effective parameters are normalized with the static mass density $\rho_{\text {steel }}=7850 \mathrm{~kg} / \mathrm{m}^{3}$ and the static Young's modulus $E_{\text {steel }}=200 \mathrm{GPa}$, respectively. These two effective 
parameters are simultaneously negative in the overlapping range $[17.6 \mathrm{kHz}-18.4 \mathrm{kHz}]$, resulting in a negative refractive index with a bandwidth of $0.8 \mathrm{kHz}$. In this overlapping band, the double negative mechanism of the single-phase chiral EMMs is that both the group velocity and the refractive index are negative, which exhibits the unique properties of the so-called left-handed materials (LHM) [6]. Note that the discrepancies of the frequency range between the effective parameters and the band structures may be attributed to the following two reasons: (1) the averaged boundary technique is accurate only in the long wavelength assumption (e.g., $\lambda \geq 20 a$ ) of the considered model [33], and (2) the global stress may be asymmetric due to the introduction of the rotational resonance, which results in the discrepancies of the averaged effective forces on the boundaries of the unit cell. Further consideration, e.g., high order continuum theories [35], with respect to the computation of the effective parameters of the chiral EMMs, is required in the future.

To further explain these unique properties of the chiral EMMs, the transmission of the longitudinal and the shear waves are calculated by using a metamaterials sample which contains $6 \times 1$ unit-cells along the $\Gamma X$ direction, as shown in Fig. 2(c). In the computation, a normal harmonic force is incident on the left side of the sample and is served as the input of the longitudinal/transverse waves. The periodic boundary conditions (PBC) are applied on the top and bottom sides of the sample. A perfect matched layer (PML) is applied in the right side of the sample to avoid the reflected waves. The transmission property of the longitudinal/transverse waves is calculated based on the ratio of the integral of the displacements along the normal/tangential directions of the output side to that of the input side. The results are plotted in Fig. 2d, where the transmissions of the longitudinal and the transverse waves are denoted as " $\mathrm{L}$ wave" and "T wave", respectively. It can be seen that the longitudinal wave can be transmitted into the sample over the frequency range $[18.4 \mathrm{kHz}-18.7 \mathrm{kHz}]$, which is very close to the resonant frequency with negative bulk modulus. Meanwhile, it is interesting to notice that the transverse wave is found to have a pass band in a very close frequency range $[18.3 \mathrm{kHz}-18.7 \mathrm{kHz}]$, although the effective shear modulus is positive over this frequency range. It is speculated that this may be caused by the 
mode conversion between the longitudinal and transverse waves [28], which will be validated in Section 5.

In order to explain the translational and rotational oscillation modes more clearly, a mass-spring system representing the motion of $\Gamma$ point is shown in Fig. 3. The translational movement of the inner core are modeled as the in-plane hybrid movement of the bending springs and tension-compression springs. The connecting ribs 2 and 4 work as the bending beams, and the ribs 1 and 3 work as the tension-compression springs in Fig. 3(a). The corresponding bending stiffness $k_{t s}$ and tensile stiffness $k_{t l}$ can be expressed by [36]: $k_{t s}=3 E I / l^{3}$ and $k_{t l}=E w / l$, where $I$ denotes the moment of inertia, $E$ represents the Young's modulus, $w$ is the width of the rib, and $l$ stands for the length. Notice that the height of the ribs is considered as a dimensionless number of 1 . Since two bending beams and two tension-compression springs are contributed to the translational oscillation, the effective longitudinal stiffness $k_{\text {leff, }}$, which is the sum of four springs, can be calculated as $k_{\text {leff }}=2 k_{t l}+2 k_{t s}$. The translational natural frequency can be approximately determined by [37]:

$$
\omega_{l}=\sqrt{\frac{k_{\text {leff }}}{m}}=\sqrt{\frac{2 E\left(3 I / l^{2}+w\right)}{m l}}
$$

where $m$ is the mass of the inner core.
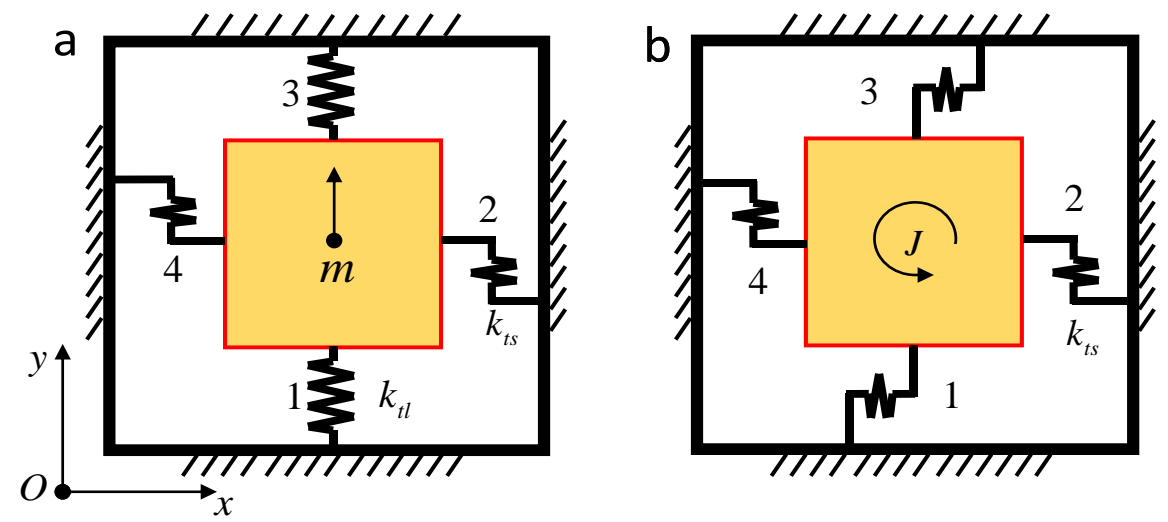

Fig. 3 The simplified mass-spring system model of (a) the translational system corresponding to S4 mode, and (b) the rotational system corresponding to S5 mode.

For the rotational oscillation mode, only the bending deformation of the connecting ribs is contributed to the rotation resonance, as shown in Fig. 3(b). Since the connecting ribs 1-4 work as the bending beams, the corresponding effective 
stiffness $k_{\text {reff }}$ can be expressed by $k_{\text {reff }}=4 k_{t s}=12 E I / l^{3}$. The rotational natural frequency can be computed by:

$$
\omega_{r}=\sqrt{\frac{k_{\text {reff }}}{J}}=\frac{2}{l} \sqrt{\frac{3 E I}{J l}}
$$

where $J$ is the rotational inertia.

By using Eqs. (11)-(12), the simultaneously negative mass density and negative bulk modulus can be realized. Tuning of the geometrical parameters results in two desired natural frequency $\omega_{r}$ or $\omega_{l}$. This scheme is an effective way to realize the desired single resonant frequency. However, randomly selecting a set of geometry parameters of Eqs. (11)-(12) are unlikely to yield double negativity of the material parameters across a broad frequency band. Thus, it is very important to propose a straightforward and feasible searching strategy to maximize the frequency range of the double negativity.

\section{Shape optimization strategy}

In this section, the objective and the constraint functions of the optimization strategy will be constructed. Firstly, the influence of the geometrical parameters on the bandwidth of double negativity is studied based on the global sensitivity analysis. Secondly, the objective and constraints formulations are presented. Finally, the procedure of the shape optimization is given.

\subsection{Influence of design variables on the bandwidth of double negativity}

It is noted that the bandwidth of double negativity is an implicit function of the geometrical parameter [8]. Therefore, an alternative way of investigating the influence of the geometrical parameters on the frequency range of double negativity is the application of global sensitivity analysis (GSA) [38, 39]. Several GSA methods have been proposed for different purposes, e.g., the variance-based GSA, the moment-independent GSA, the qualitative GSA, the non-parametric methods, and so on $[40,41]$.

In this paper, a non-parametric method $[38,41]$ using the regression-based techniques is applied. Specifically, a typical test with $N=2000$ samplings is firstly 
performed to provide a general basis for both the evaluation of the frequency bandwidth and the evaluation of the effects of independent parameters. The samplings are generated randomly in a predefined bound with a uniform distribution function. The bounds are given in Tab. 2 .

Tab. 2 Geometry parameters of the shape

\begin{tabular}{cccc}
\hline \multirow{2}{*}{ Parameters } & \multirow{2}{*}{$\begin{array}{c}\text { Point for presenting } \\
\text { the interaction effect }\end{array}$} & Lower bound & Upper bound \\
\hline$b$ & 30 & 28 & 31.8 \\
$c$ & 27 & 24 & 29 \\
$d$ & 8 & 7 & 10 \\
$h$ & 11 & 4 & 12 \\
$\theta$ (degree) & 10 & 0 & 45 \\
$w$ & 1 & 0.6 & 2 \\
\hline
\end{tabular}

Specifically, the typical sampling is defined as:

$$
\mathbf{X}_{i}=[b, c, d, h, \theta, w], i=1,2, \ldots, N
$$

Then the evaluations of the frequency bandwidth of the double negativity are calculated by using the effective medium approach mentioned in Section 2.1. In these evaluations, the target frequency range is defined as $[0-20 \mathrm{kHz}]$.

Besides, to construct a relationship between the design variables and the bandwidth, the radial basis function (RBF) is applied to the regression analysis [39]. The RBF model is defined as:

$$
Y(\mathbf{X})=\sum_{i=1}^{N} \eta_{i} \psi\left(\left\|\mathbf{X}-\mathbf{X}_{i}\right\|\right), i=1,2, \ldots, N
$$

where $\mathbf{X}_{i}$ is the design variable vector with six-dimensional, and $Y$ stands for the approximated value of the objective function. $\psi$ denotes the basis function, and $\eta$ stands for the weight of basis function derived through fitting the input-output model to the training data. Note that the operator $\|\cdot\|$ measures the Euclidean distance between two sampling points. Applying the training operation for Eq. (14) yields a linear system of equation:

$$
\mathbf{Y}=\boldsymbol{\eta}
$$

where $\mathbf{Y}$ denotes the output vector, and $\boldsymbol{\eta}$ is the Gramian matrix of design variables. $\boldsymbol{\psi}$ 
represents the vector of basis function weights, which can be determined by:

$$
\boldsymbol{\psi}=\left[\begin{array}{cccc}
\psi\left(\mathbf{X}_{1}, \mathbf{X}_{1}\right) & \psi\left(\mathbf{X}_{1}, \mathbf{X}_{2}\right) & \ldots & \psi\left(\mathbf{X}_{1}, \mathbf{X}_{N H}\right) \\
\psi\left(\mathbf{X}_{2}, \mathbf{X}_{1}\right) & \psi\left(\mathbf{X}_{2}, \mathbf{X}_{2}\right) & \ldots & \psi\left(\mathbf{X}_{2}, \mathbf{X}_{N H}\right) \\
\vdots & \vdots & \ddots & \vdots \\
\psi\left(\mathbf{X}_{N H}, \mathbf{X}_{1}\right) & \psi\left(\mathbf{X}_{N H}, \mathbf{X}_{2}\right) & \ldots & \psi\left(\mathbf{X}_{N H}, \mathbf{X}_{N H}\right)
\end{array}\right]
$$

where $H$ is the number of dimensions of $\mathbf{Y}$. In this section, the Gaussian function defined by $\psi=\exp \left(-\left\|\mathbf{X}-\mathbf{X}_{i}\right\|^{2} / 2 \delta^{2}\right)$ is served as the radial basis function, in which $\delta$ represents the predetermined RBF parameter.
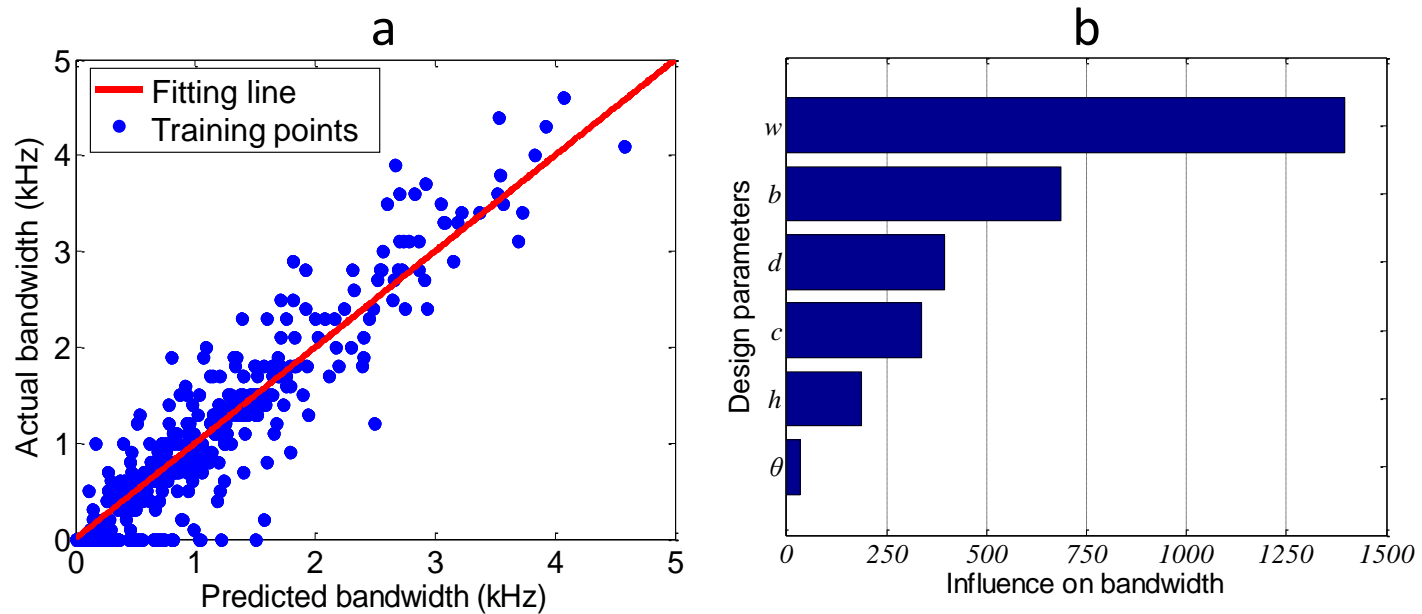

Fig. 4 The metamodel for the bandwidth fitting: (a) the curve of predicted bandwidth against the actual bandwidth, and (b) the global influence of the design parameters (average gradient) on the bandwidth.

Figure 4(a) presents the fitting of the bandwidth after the training test. To assess the accuracy of the fitting metamodel, the index of R-square is adopted [39]. In this fitting, the value of R-square is 0.8843 (the maximum is 1 and the acceptance level is 0.8), which indicates that the bandwidth can be predicted successfully by using the constructed metamodel. Then, the influence of the parameters on the bandwidth is computed and shown in Fig. 4(b), where the information of the average gradient of each geometry parameter is plotted. It implies that the parameter $w$ is the most sensitive factor that influences the width of the frequency range, and the parameter $\theta$ is the least sensitive parameter. In order to exhibit the interaction effect of the six geometry parameters, the contour graphs of bandwidth versus any two independent parameters at point $\mathbf{X}=[30,27,8,11,10,1]^{\mathrm{T}}$ are presented in Fig. 5, where a higher level 

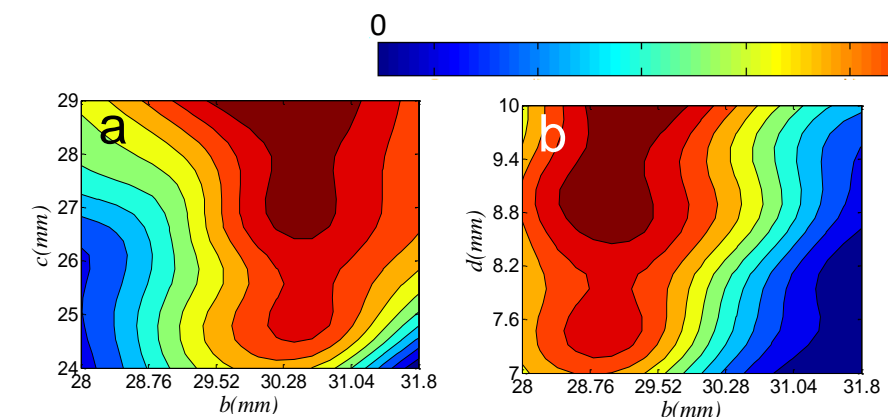

$\max$
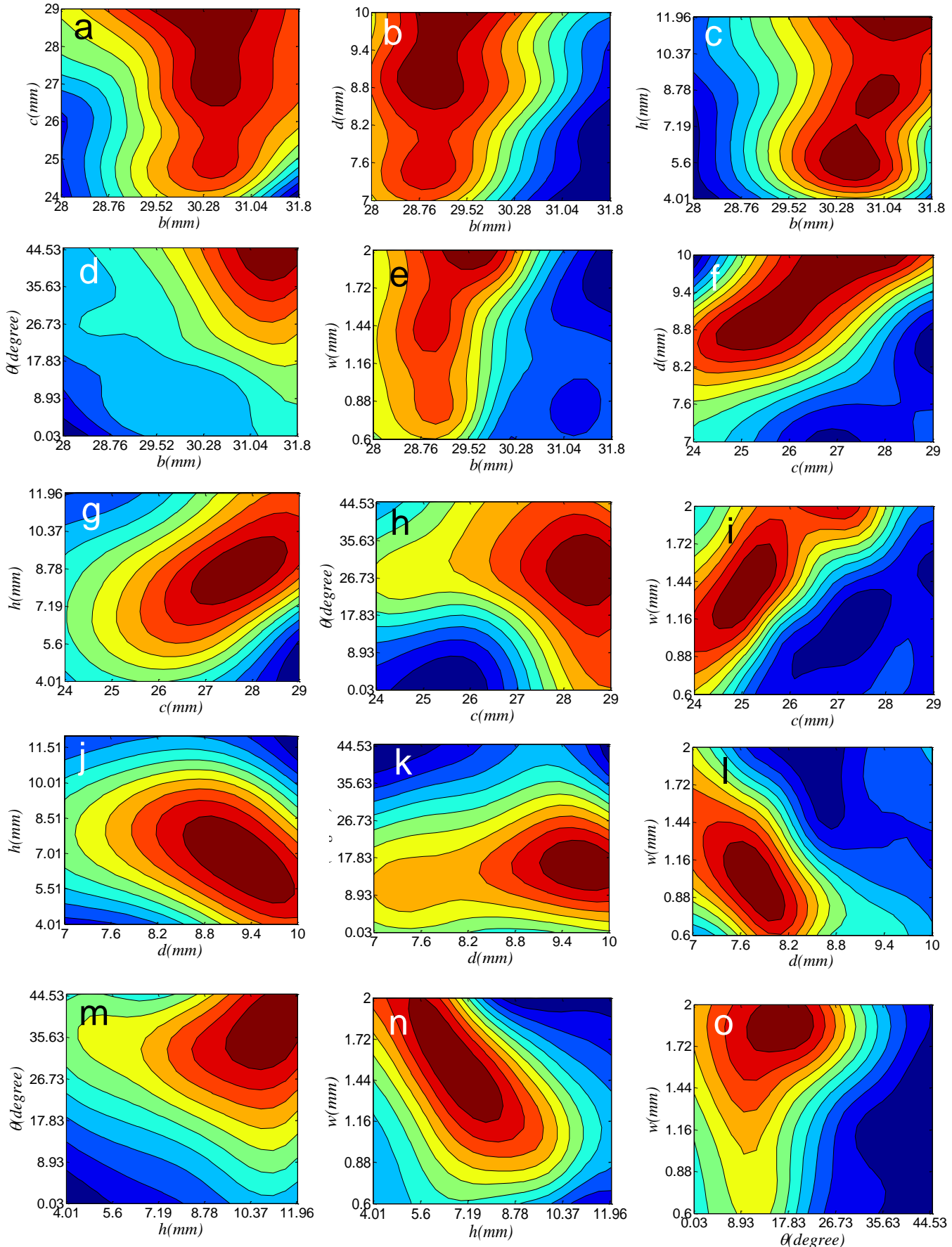

Fig. 5 The interaction effects map of the design variables on the frequency bandwidth.

of red color denotes a broader frequency range of double negativity. It can be observed that a different combination of the geometry parameters can lead to a different width of the frequency ranges of double negativity. For instance, Fig.5(a) shows that the parameter $b$ with a moderate level and the parameter $c$ with a high level are contributed to enlarging the bandwidth of the double negativity, while a high 
level of parameter $b$ accompanied with a high level $\theta$ is helpful to maximize the bandwidth in Fig. 5(d).

\subsection{Optimization formulation}

In this subsection, the optimization strategy is constructed. Firstly, it is necessary to understand the evolution history of the double negativity ( $\rho_{\text {eff }}$ and $\left.K_{\text {eff }}\right)$ when the geometrical parameters are updated iteratively. To be more clearly, an example with $\mathbf{X}=[30,27,2.5,11,0,1]^{\mathrm{T}}$ is discussed. The effective parameters are plotted in Fig. 6 .

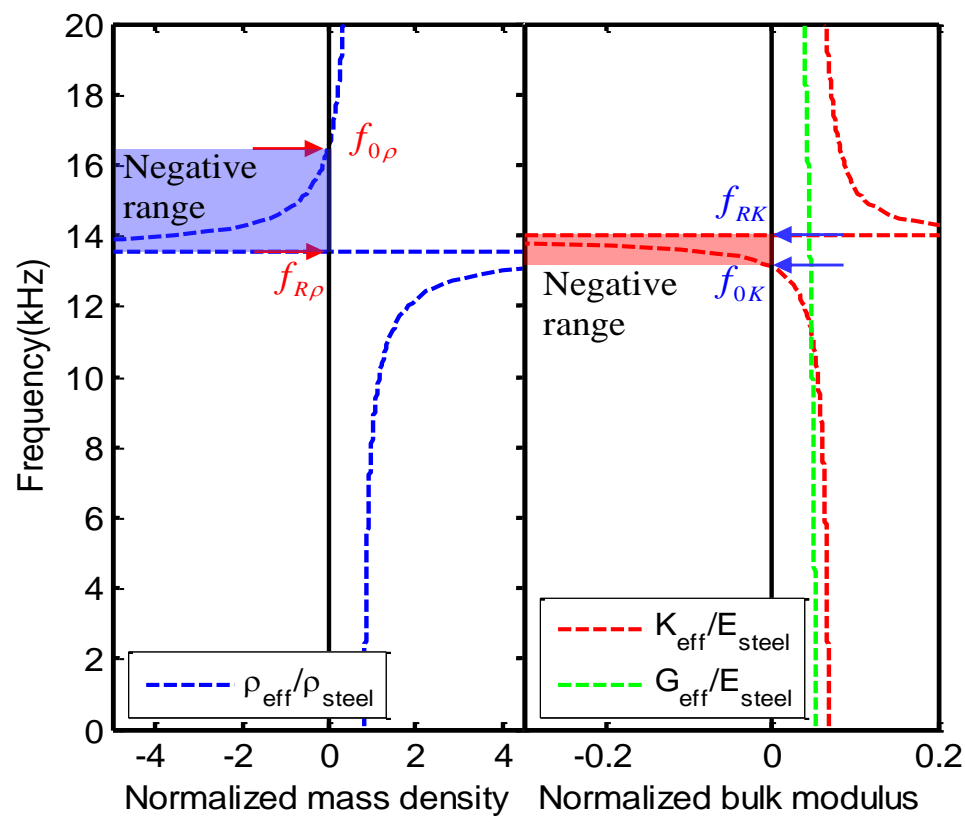

Fig. 6 The negative properties curve as a function of frequency.

It can be seen from Fig. 6 that the effective mass density is a constant in the lower frequency range. As the frequency increases, the effective mass density reaches its maximum at the resonant frequency $f_{R \rho}$. Then it changes sharply to the negative infinity. In the negative range, the effective mass density increases from the global minimum to a positive value at frequency $f_{0 \rho}$. Finally, the effective mass density increases from this frequency and stops at the end frequency $f_{\max }$. On the other hand, the curve of the effective bulk modulus is different. It is a constant at the low frequency range, and reaches zero at the frequency $f_{0 K}$. The effective bulk modulus then drops to a negative infinity and dramatically goes up to the positive maximum at the resonant frequency $f_{R K}$. Above this frequency, the effective bulk modulus gradually decreases from the positive maximum to a positive value. Meanwhile, the effective shear modulus 
is positive in the considered frequency range.

It can be found from the above two curves that four frequency points including $f_{0 \rho}, f_{R \rho}, f_{0 K}$, and $f_{R K}$ (as denoted by the red and blue arrows) are very important, which can be used to distinguish the positive value and the negative value of the effective parameters. As a result, the following inequations can be derived $f_{0 \rho}>f_{R \rho}$ and $f_{R K}>f_{0 K}$, if both monopolar and dipolar resonance take place. Thus, a straightforward way to generate a frequency band of double negativity is firstly to ensure the inequation $\min \left\{f_{0 \rho}, f_{R K}\right\}>\max \left\{f_{R \rho}, f_{0 K}\right\}$. Secondly, the optimization scheme should be further designed to enlarge the bandwidth of that frequency range.

Another important consideration in the shape optimization is the location of the frequency range of the double negativity. Hence, a scatterplot between the bandwidth and its location is displayed in Fig. 7. Here, the midfrequency of the bandwidth represents the location in the target frequency range. These sampling points are the same as those used to carry out the regression analysis in section 3.1. Fig. 7 shows that a broader frequency range of double negativity always locates above half of the cutoff frequency (Here is $10 \mathrm{kHz}$ ), which implies that a broader and a higher frequency range of double negativity may be conflicting. This should be taken into consideration in the construction of the optimization formulation.

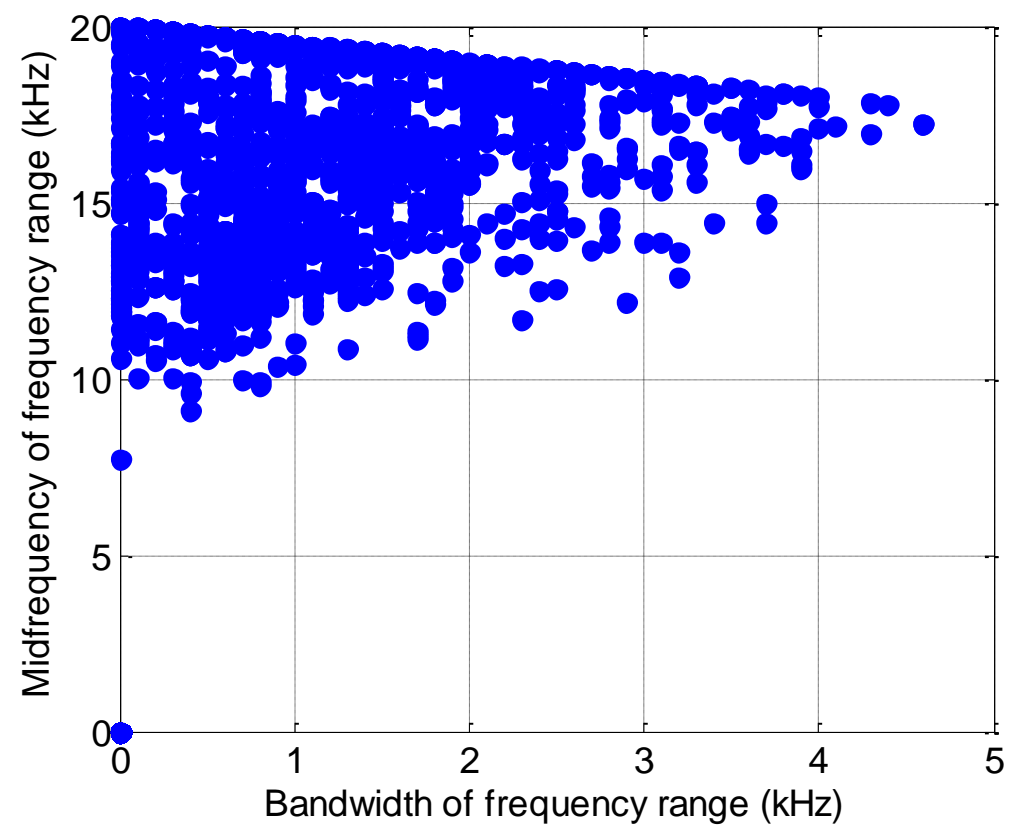

Fig.7 Scattered 2D plot between the midfrequency and bandwidth of the frequency range of double 
negativity.

It is noted that in Ref. [8], a lower frequency range of double negative properties is much more preferred than a higher frequency range. However, this is different in the present optimization, as demonstrated in Section 3.1, to obtain a chiral EMM which possesses a lager bandwidth as well as a lower location of the frequency range of double negativity is very challenging. These two objectives may be conflicting during the shape optimization process. In this case, given an original chiral EMM, the main purpose of the optimization approach is to find an optimized chiral EMM that has the largest double negativity band within a specified relative frequency range. Without loss of generality, the longitudinal waves are assumed to propagate along the $\Gamma \mathrm{X}$-direction $[14,28]$, and the optimization strategy is focused on this direction. The shape optimization problem can be mathematically written as:

For: $f_{\min } \rightarrow f_{\max }$

Find: $\mathbf{X}=[b, c, d, h, \theta, w]^{\mathrm{T}}$

Maximize: $F(\mathbf{X})=F D \cdot\left\{\max _{\forall l \in(1,2, \ldots, L)}\left[f_{\rho_{\text {eff }}^{-}(l), K_{\text {eff }}^{-}(l)}\right]-\min _{\forall l \in(1,2, \ldots, L)}\left[f_{\rho_{\text {eff }}^{-}(l), K_{e f f}^{-}(l)}\right]\right\}$

where $\mathbf{X}$ is the design variable, and $F(\mathbf{X})$ denotes the objective function. $F D$ is the number of frequencies when the negative $\rho_{\text {eff }}$ and $K_{\text {eff }}$ are generated simultaneously, and $f_{\rho_{e f f}^{-}(l), K_{e f f}^{-}(l)}$ declares the corresponding frequencies ( $l$ represents the serial number).

Note that the frequency step size of the searching strategy within the target frequency range is defined as $\Delta f=0.1 \mathrm{kHz}$, and $L$ represents the total number of the frequencies between $f_{\min }$ and $f_{\max }$. In the iteration procedure, both the values of $F D$ and $F(\mathbf{X})$ are expected to increase sharply once the double negativity is generated, which guides the optimized strategy to explore more frequencies.

In order to find a feasible solution, the constraints are still to be established. Generally, for the purpose of generating a chiral EMM which can be well manufactured and discretized, the structure gap (white area of the structure in Fig. 1a) and the width of the connecting beam have to be assigned a smallest size during the iteration. [42]. To be more specific, the mesh-refinement is always required in order to 
obtain a better modeling and description of the boundaries [42]. However, a mesh-refinement may result in a huge computational burden in the finite element modeling. To compromise these two issues, the smallest width of the connecting beam is suggested to be larger than a threshold. It has been tested that the width $1 \mathrm{~mm}$ works well for the optimization. Then the constraints are defined by:

$$
\begin{array}{ll}
\text { Subject to: } & b-c \geq 1 \mathrm{~mm} \\
& c / 2-(d+2 w) \geq 1 \mathrm{~mm}
\end{array}
$$

\subsection{Procedure for shape optimization}

As shown in Fig. 4(a), the condition in which a metamodel can be used for global sensitivity analysis is that this metamodel is very accurate, which requires a huge number of samplings that cover the global design space. Besides, it is noted that although a metamodel is accurate with acceptable accuracy, it is not necessary to acquire the best values of the objective function [43]. Because it is assumed that the design parameters and the bandwidth sample can be well fitted by a linear, continuous and smooth regression model during the construction of the metamodel. Nevertheless, these assumptions are not always satisfied. Thus, a suitable optimization strategy is required to enlarge the bandwidth of double negativity. To this end, the single-objective genetic algorithm (GA) is selected as the optimizer [10, 44, 45], which can carry out a feasible solution of Eqs. (17)-(18). Based on the survival-of-the-fittest principle to simulate the natural selection, the GA has been increasingly applied in the design of EMMs. In the GA optimization problem, the design variable is considered as a chromosome which possesses a number of genes describing the geometry of the chiral EMMs. And the fitness function of each structure is computed to determine whether these genes require a further gene operation. By repeating this process, a number of excellent individuals can be generated, and the structure is updated iteratively until the stop criteria are satisfied.

Remark 2: It should be noted here that the GA is not the only optimizer that can be used for the current optimization problem. One is free to choose the preferred optimizers, since the common goal is to enlarge the original double negativity 
frequency band.

In this work, the GA procedure for the shape optimization scheme is displayed in

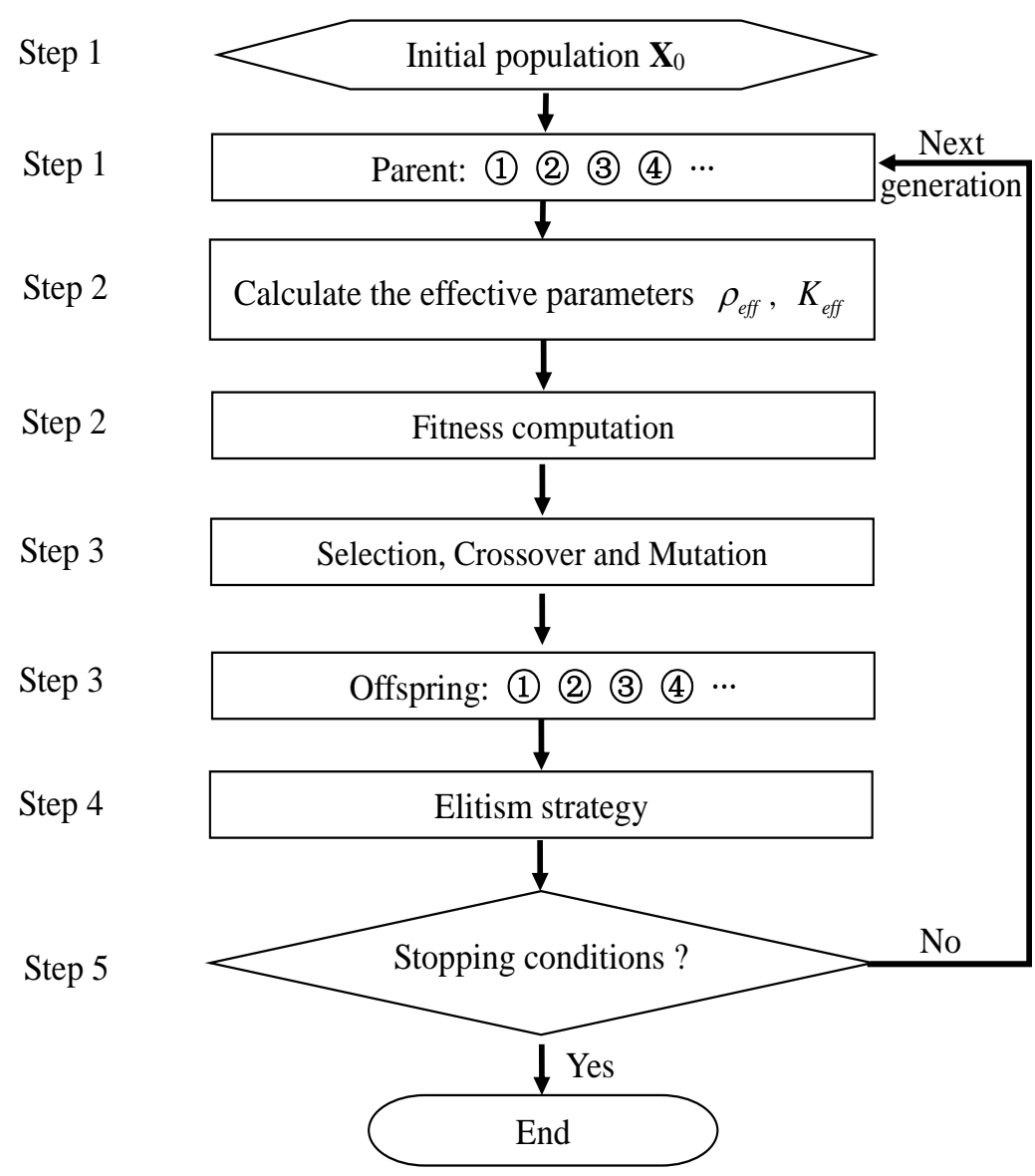

Fig. 8 The flowchart of the implementation of the shape optimization procedure [8].

Fig. 8 and summarized as follows:

Step 1: The GA algorithms begins with an initial population (where the population size is prescribed as $N_{p}$ ).

Step 2: For each parent generated in step 1, compute the fitness function. In this paper, the fitness function is defined from the objective function (Eq. (17)) with the penalty effect in order to obtain a good convergence during the optimization. The transformed fitness function is thus expressed by:

$$
\text { Minimize: fitness }(\mathbf{X})=-F(\mathbf{X})+N_{\rho}(L-m)+N_{K}(L-n)
$$

where $m$ and $n$ represent the serial numbers of the frequencies as the double negative parameters $\rho_{\text {eff }}^{-}$and $K_{\text {eff }}^{-}$emerge, respectively; $N_{\rho}$ and $N_{K}$ are two positive penalty coefficients. 
Steps 3-4: Apply the tournament selection, crossover, and mutation operations to the current generation; adopt the elitism strategy to the optimization process.

Step 5: Check the convergence whether the stopping conditions are satisfied, if no, repeat steps 1-4.

\section{Numerical examples}

In this section, three examples will be conducted to demonstrate the effectiveness of the shape optimization strategy. The first example deals with the optimization with different initial shapes. In the second one, shape optimization with a lower target frequency range is investigated. The third example focuses on shape optimization with more design variables. Unless otherwise stated, the settings used in GA will be the same. The population size is prescribed as $N_{p}=30$, the elite count is $N_{e}=4$, the crossover fraction is $N_{c}=0.8$, and the mutation rate is $N_{m}=0.01$. The penalty coefficients in Eq. (19) are $N_{\rho}=1$ and $N_{K}=1$. In all these examples, the number of fitness function evaluations is the product of the generation number and the population size, and the frequency step size of the searching strategy within the target frequency range is defined as $0.1 \mathrm{kHz}$. Besides, the stop criteria of the GA optimization are defined as the tolerance of the fitness function and the number of the stall generations, i.e., the value of tolerance is $1 \mathrm{e}-6$ and the number of the stall generations is 40 . It has been tested that with the stop criteria the frequency band exhibiting double negativity can be optimized to a largest value within a target frequency range.

\section{Case 1: optimization with different initial shapes}

In this subsection, four types of initial shape are considered, as shown in Fig. 9. Note that the circular frame means that the inner shape of the frame is circular and the radius is denoted as $r_{2}$, whereas its outer shape is still the square shape, which can be seen in Fig. 9(c-d). In Fig. 9(b), the radius of the circular core is defined as $r_{1}$. The lattice constants are all considered as $a=0.045 m$, and the target frequency range is $[0-20 \mathrm{kHz}]$. Note that the longitudinal wave velocity is determined by $c_{l}=\sqrt{E(1-v) /[\rho(1+v)(1-2 v)]}=5856.4 m / s$. For the considered examples, the shortest 
wavelength at frequency $20 \mathrm{kHz}$ is expressed as $\lambda \approx 6.5 a$. The initial shape is expressed as $b=30, c=27, d=8, h=11, \theta=0$, and $w=1$, and the bounds of the design variables are defined in Tab. 1. The constraints shown in Eq. (18) are adopted. Note that the initial shape of the chiral EMM possesses a double negative bandwidth of $1.9 \mathrm{kHz}$, where the effective mass density is negative in $[16.3-19.6 \mathrm{kHz}]$, and the effective bulk modulus exhibits negative property in [17.3-19.2kHz].
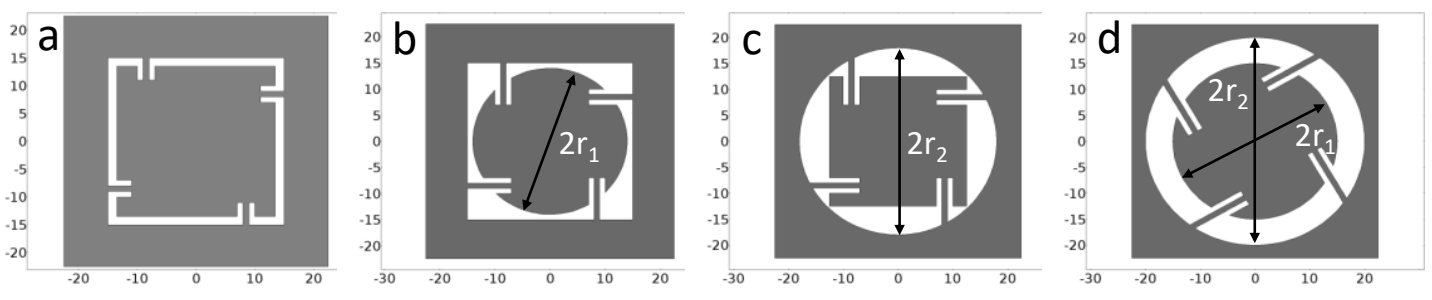

Fig. 9 Four types of initial shapes of chiral EMMs: (a) square core with a square frame, (b) circular core with a square frame, (c) square core with a circular frame and (d) circular core with a circular frame.

Figure 10 shows the shapes and corresponding frequency range of the effective material parameters of the optimized structures. The resulting frequency band of the negative mass density of the optimized EMMs is [14.7-19.7 kHz], and the frequency range of negative bulk modulus is [14.1-19.9 kHz]. This leads to a simultaneously negative frequency range of $5.0 \mathrm{kHz}$, which is arisen from $14.7 \mathrm{kHz}$ and ended at $19.7 \mathrm{kHz}$, indicating the bandwidth of double negativity is found to be extended. The predicted overlapped negative mass density and negative bulk modulus frequency range matches the band diagram very well, which is highlighted Fig. 10b. Meanwhile, the effective shear modulus is always positive in the considered frequency range. With the effective bulk modulus and the effective shear modulus, the effective Young's modulus, which is determined by $E_{\text {eff }}=K_{\text {eff }}+G_{\text {eff }}$, is found to be negative in the frequency range [16.1-19.7 kHz]. This frequency range agrees very well with the transmission property when the longitudinal wave impinging to the sample, see Fig. $10 \mathrm{c}$ and $10 \mathrm{~d}$. It is interesting to notice that the transverse wave can also be transmitted over the frequency range with negative Young's modulus. In this situation, it is speculated that this may be caused by the mode conversion between the longitudinal and transverse waves. This phenomenon is very similar to those found in the work [28], and it will be validated in the next section. Fig. 11 displays the evolutionary 
history of the optimization process. It can be seen that the fitness function becomes flat after nine iterations, and the iteration stops at the 50th generation. This demonstrates that the proposed methodology is able to generate a largest bandwidth with double negativity.

For other types of chiral EMMs, their shape optimizations have also been tested, but their frequency bandwidths of double negativity are found to be inferior to the first one (type (a)). Since their optimization processes are very close, only the comparison of the frequency ranges with double negativity of the optimized results is presented in Tab. 3. It is observed that both the core shape and frame shape can affect the width of the double negative frequency range. The widening of the negative bulk modulus frequency range is due to the downward movement of the start frequency in the frequency bound, whereas the widening of frequency of the negative mass density is attributed to the upward movement of the end frequency of the frequency bound. This implies that the structures with square frame (types (a) and (b)) can result in an easier rotational mode in a lower frequency range and thus provide a very wider frequency range for the monopole resonance. The structures with circular frame, i.e., types (c) and (d), can generate an easier translational mode in a higher frequency range and therefore provide a wider frequency range for the dipole resonance. Among the four types of optimized EMMs, type (a) gives the largest frequency range for double negativity.

Note that the presented optimization strategy is distinct from the one proposed in Ref. [8]. The goal of the latter one is to maximize the frequency range of double negativity by using topology optimization. It shows that both the negative bulk modulus and negative mass density are generated through the rotational resonance of the four central lumps. For the purpose of comparison, the optimization strategy of this paper is based on the monopolar and dipolar resonance of the central single lump. The main objective is to capture the optimized shape in order to generate a broad frequency range in which the monopolar and dipolar resonance can be produced simultaneously. 

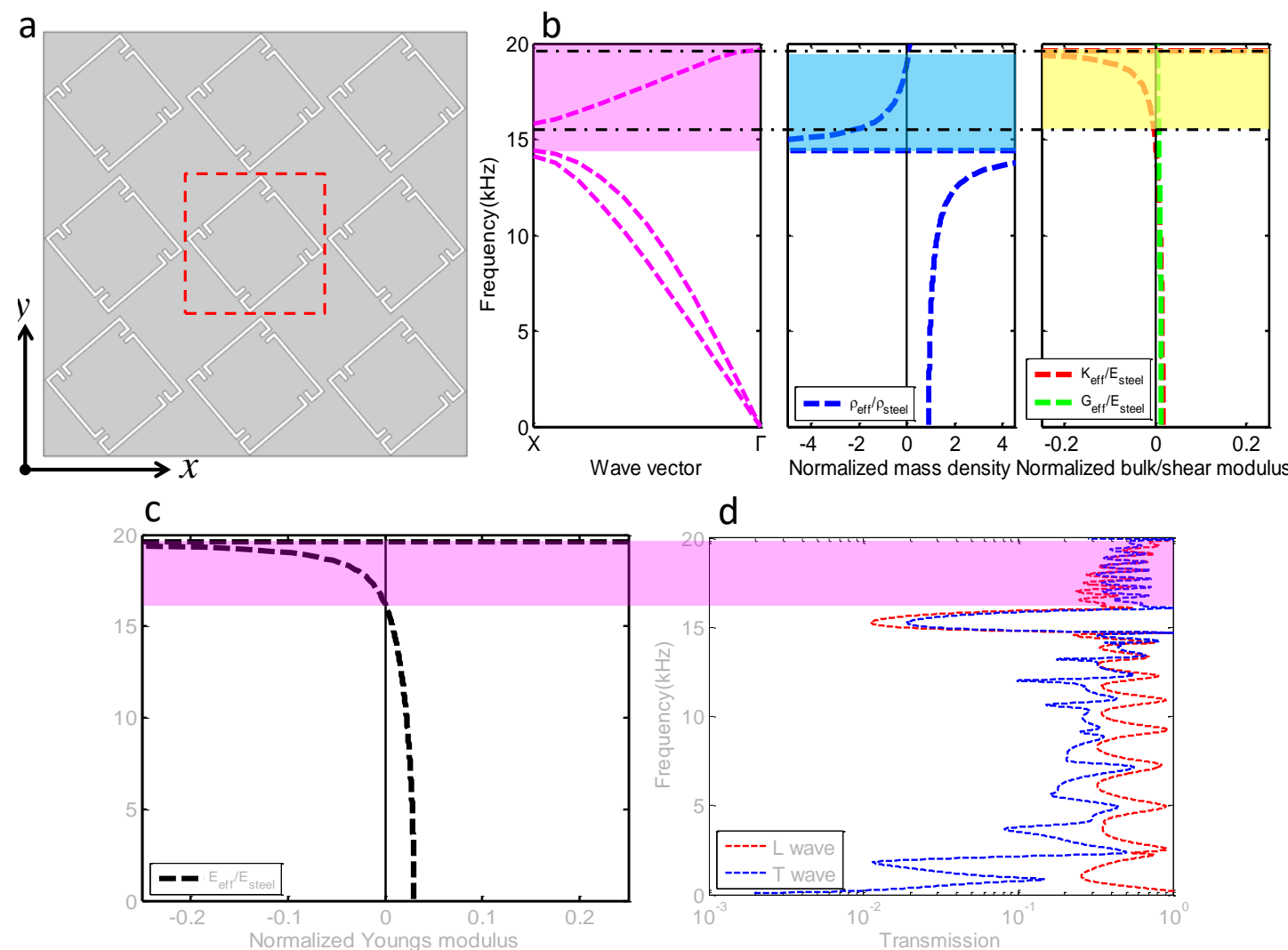

$\mathrm{d}$

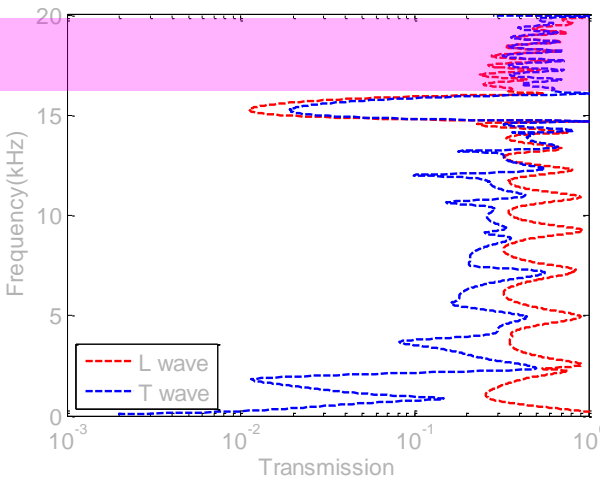

Fig. 10 Effective material parameters, the dispersion curves and the transmission property of the optimized chiral EMMs. (a) the optimized EMMs with $3 \times 3$ unit cells; (b) the double negative parameters and the dispersion curves along $\Gamma X$ direction; (c) the effective Young's modulus, and (d) the transmission property when the longitudinal wave impinges to the sample.

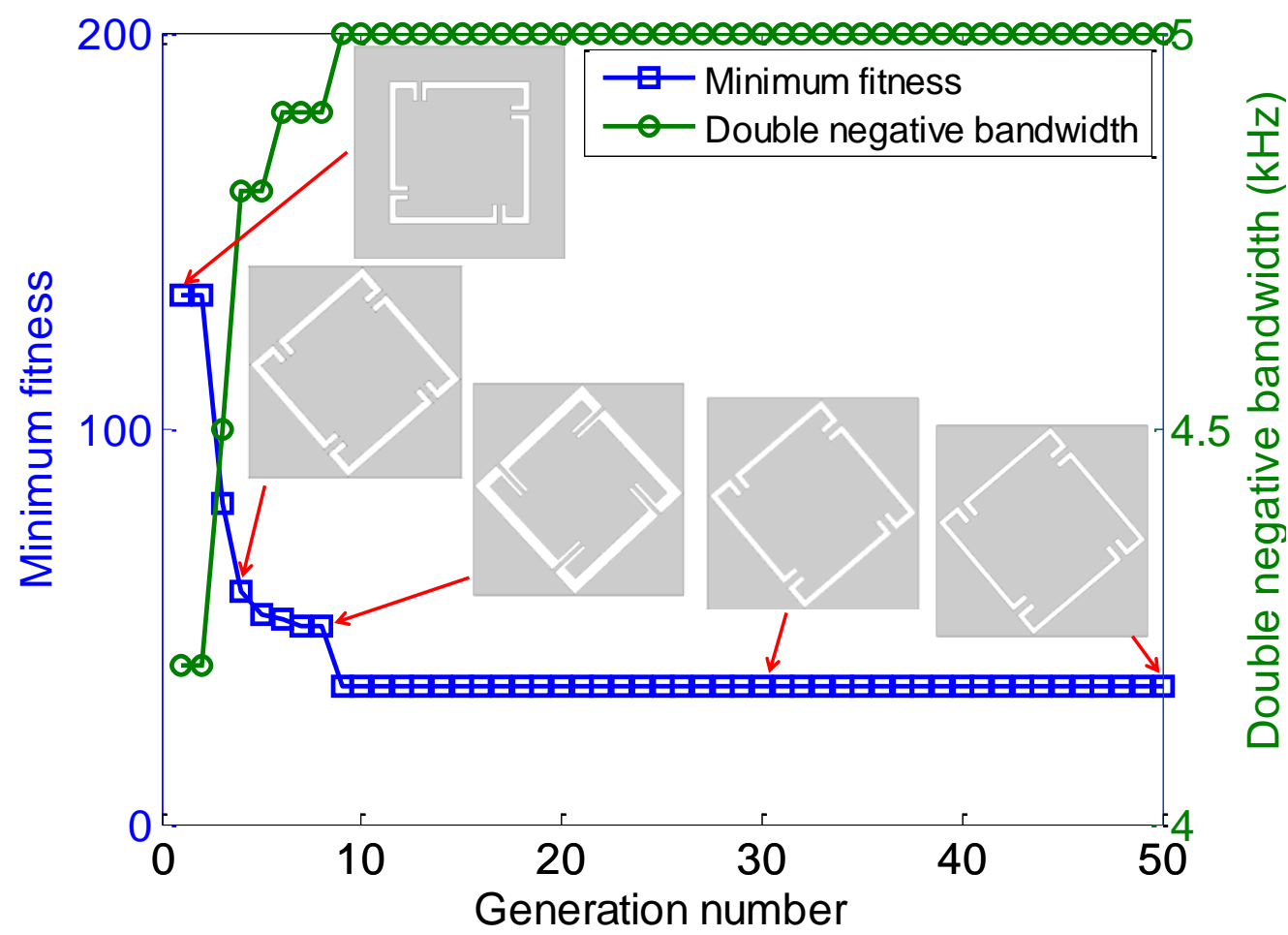


Fig. 11 The shape and the double negative bandwidth evolution with the increase of the generation number.

Tab. 3 Comparison of the double negativity frequency range for four types of chiral EMMs

\begin{tabular}{cccc}
\hline Type & $\begin{array}{c}\text { Double negative } \\
\text { frequency range }\end{array}$ & $\begin{array}{c}\text { Double negativity } \\
\text { bandwidth }\end{array}$ & Optimized parameters \\
\hline (a) & {$[14.7-19.7 \mathrm{kHz}]$} & $5.0 \mathrm{kHz}$ & $\mathbf{X}=[31.35,29.09,9.05,11.59,40.22,1.21]^{\mathrm{T}}$ \\
(b) & {$[13.7-17.6 \mathrm{kHz}]$} & $3.9 \mathrm{kHz}$ & $\mathbf{X}=[31.76,14.20,8.52,8.90,37.05,1.14]^{\mathrm{T}}$ \\
(c) & {$[17.0-20.0 \mathrm{kHz}]$} & $3.0 \mathrm{kHz}$ & $\mathbf{X}=[19.85,25.14,8.14,11.91,39.93,1.17]^{\mathrm{T}}$ \\
(d) & {$[16.4-20.0 \mathrm{kHz}]$} & $3.6 \mathrm{kHz}$ & $\mathbf{X}=[19.97,15.43,8.51,9.14,29.74,1.68]^{\mathrm{T}}$ \\
\hline
\end{tabular}

\section{Case 2: optimization for a lower relative frequency range}

In order to test the effectiveness of the proposed optimization approach at a smaller relative frequency, the shape optimization with a lower target frequency range is studied. Under this circumstance, the lattice constant is the same as the previous numerical case, which is fixed at $a=0.045 \mathrm{~m}$, and the target frequency range is selected as $[0-10 \mathrm{kHz}]$. Note that the propagating wavelength of the longitudinal wave in this regime is far larger than the lattice constant, which can be expressed as $\lambda \approx 13 a$. The initial shape and the bounds of the design variables are given in Tab. 4. The constraints applied in this example are similar to the previous ones.

Tab. 4 The initial point and scale of the geometry parameters of the unit cell

\begin{tabular}{cccc}
\hline \multicolumn{2}{c}{ Geometry parameters $(\mathrm{mm})$} & \multicolumn{3}{c}{ Scale } \\
\hline Variables & Initial population & Lower bound & Upper bound \\
\hline$b$ & 38 & 28 & 38.5 \\
$c$ & 27 & 24 & 29 \\
$d$ & 8 & 7 & 10 \\
$h$ & 3 & 3 & 12 \\
$\theta$ (degree) & 10 & -10 & 10 \\
$w$ & 1 & 0.5 & 2 \\
\hline
\end{tabular}

It is noted that the bounds of the design variables are changed compared with those shown in the first numerical case. The reason for this is because when the chiral topology shape of the EMMs is fixed, the definition and the selection of the parameter bounds of the design variable are very important. In the optimization experiment, it has been tested that if the original bounds (which are defined in Tab. 2) of the design variables are still applied in the current case, the frequency range with double negativity is almost zero. The optimized chiral structure with the design variable 
$\mathbf{X}=[38.46,27.26,9.15,3.00,9.98,0.50]^{\mathrm{T}}$ is shown in Figs. 12 and 13, where the effective parameters, the band curves, and the transmission property are also expressed. It can be seen that the double negative frequency range is widened from $0.8 \mathrm{kHz}$ to $1.4 \mathrm{kHz}$. This improvement is yielded by moving the lower bound of the double negativity frequency range to a lower frequency, whereas the upper bound is almost constant with a stable frequency of $f_{\max }=10 \mathrm{kHz}$. The predicted overlapped frequency band with negative mass density and negative bulk modulus is very close to the band diagram, which is highlighted Fig. 12b. Besides, the frequency ranges of the negative effective Young's modulus matches to those of the pass band of the longitudinal wave. In this example, it is found that the transverse wave is allowed to transfer in the frequency range with negative effective Young's modulus. This phenomenon is very similar to the first number example, which may be caused by the mode conversion. Fig. 13 presents the evolution histories of the fitness function and the bandwidth of double negative parameters. It shows that the minimum fitness function becomes flat after 10 generations. It can be found from Figs. 11 and 13 that searching a frequency band with double negativity at a relatively smaller frequency is more challenging. Fortunately, the proposed strategy is able to generate a frequency band with double negativity when considering a lower target frequency range, although the double negative bandwidth is smaller than those obtained from the first numerical example. 

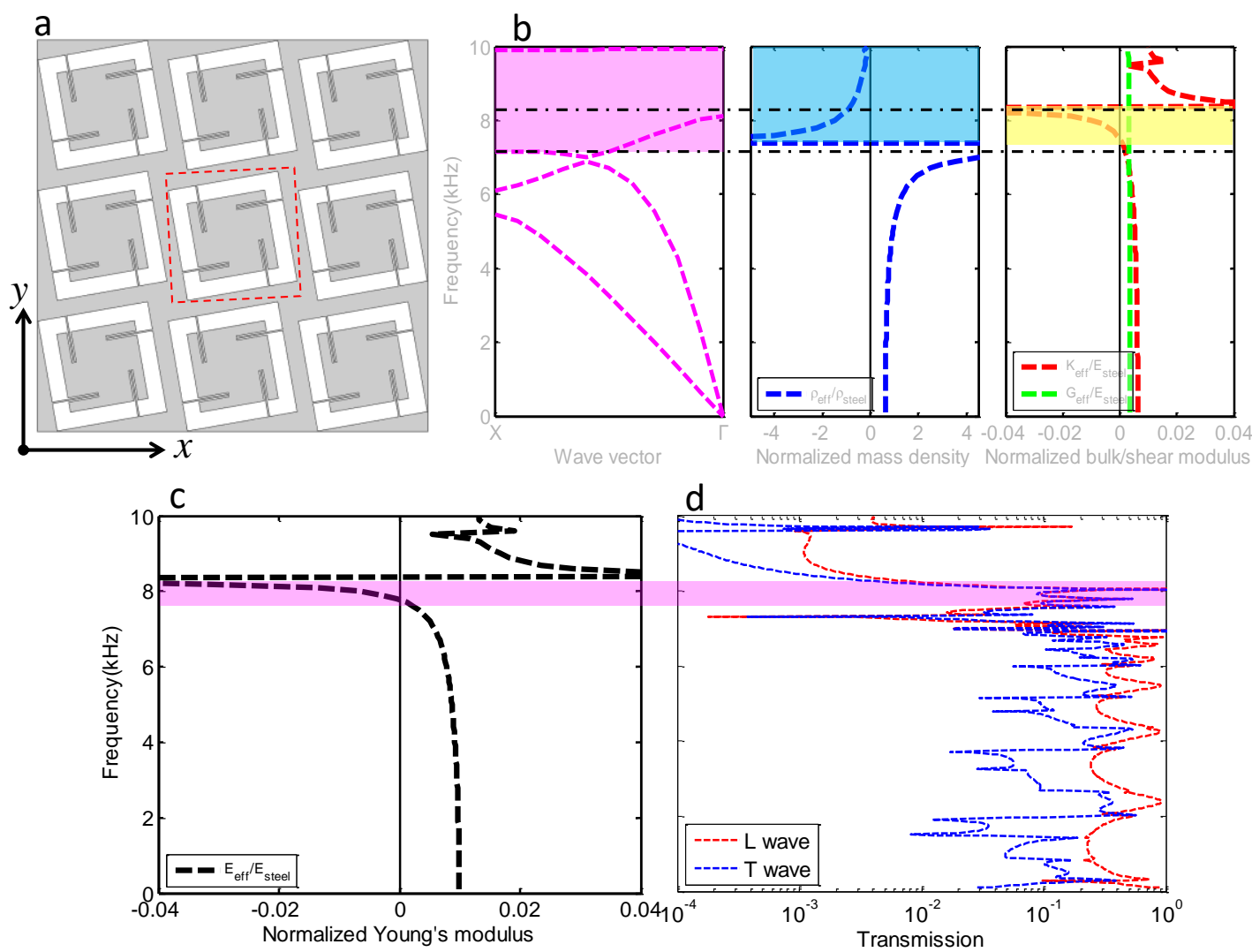

Fig. 12 Effective material parameters, the dispersion curves and the transmission property of the optimized chiral EMMs when considering a lower relative frequency range. (a) the optimized EMMs with $3 \times 3$ unit cells; (b) the double negative parameters and the dispersion curves along $\Gamma \mathrm{X}$ direction;

(c) the effective Young's modulus, and (d) the transmission property when the longitudinal wave impinges to the sample.

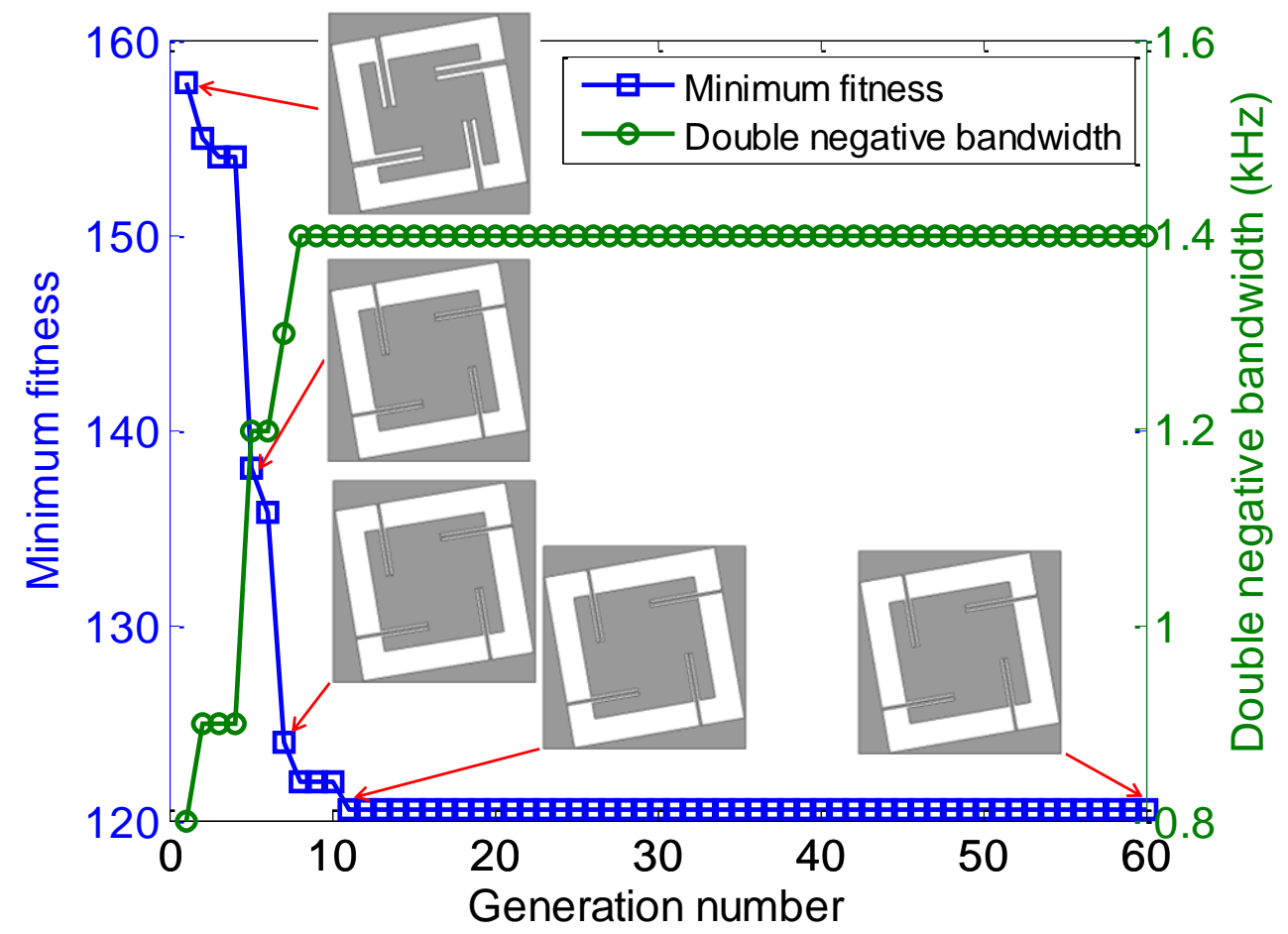


Fig. 13 The shape and the double negative bandwidth evolution with the increase of the generation number considering a target frequency range of $[0-10 \mathrm{kHz}]$.

\section{Case 3: optimization with more variables}

In this example, the shape optimization with eight variables is investigated. As shown in Fig. 14, the radius $\left(r_{3}\right)$ of the built-in circle of the inner core and the radius $\left(r_{4}\right)$ of a quarter circle of the frame are considered as the other design variables, except those expressed in Tab. 1. The lattice constant $a$ is $0.045 m$, and the target frequency range is $[0-20 \mathrm{kHz}]$. The constraints used in GA are still the same as those in case 1. Besides, the bounds for $r_{3}$ and $r_{4}$ are expressed as $0.5 \mathrm{~mm} \leq r_{3} \leq 8 \mathrm{~mm}$ and $0.5 \mathrm{~mm} \leq r_{4} \leq 8 \mathrm{~mm}$. Note that these two variables are independent of each other. The design variable for this case is defined as: $\mathbf{X}=\left[b, c, d, h, \theta, w, r_{3}, r_{4}\right]^{\mathrm{T}}$.

Figures 14 and 15 show the effective parameters and the band curves of optimized shapes. The size of the optimized structure is $\mathbf{X}=[31.57,28.35,9.98,11.28,37.39,1.09,3.96,4.84]^{\mathrm{T}}$. It can be observed that the frequency range of double negativity is enlarged from $2.4 \mathrm{kHz}$ to $5.0 \mathrm{kHz}$. This optimized frequency band is almost the same as the optimization with six design variables (case 1 type (a)). Besides, the effective parameters match very well with the band structures, and the transmissions of the longitudinal and the transverse waves inside the chiral EMMs are shown in Fig. 14d, which agrees well with the frequency range with negative effective Young's modulus. Again, it is found that over this frequency range the transverse waves are allowed to pass the chiral EMMs, as a result of mode conversion. In this numerical case, more materials of this chiral EMM are deleted compared with the first numerical case, which implies that the proposed strategy can obtain an identical broad frequency band with a lighter weight. The evolutionary history of the optimization process is plotted in Fig. 15, where the fitness function stops at $20^{\text {th }}$ generation. Comparing with the optimization process of type (a), it indicates that the proposed optimization method is capable of enlarging the frequency band of the double negativity when two more design variables are taken into consideration. 

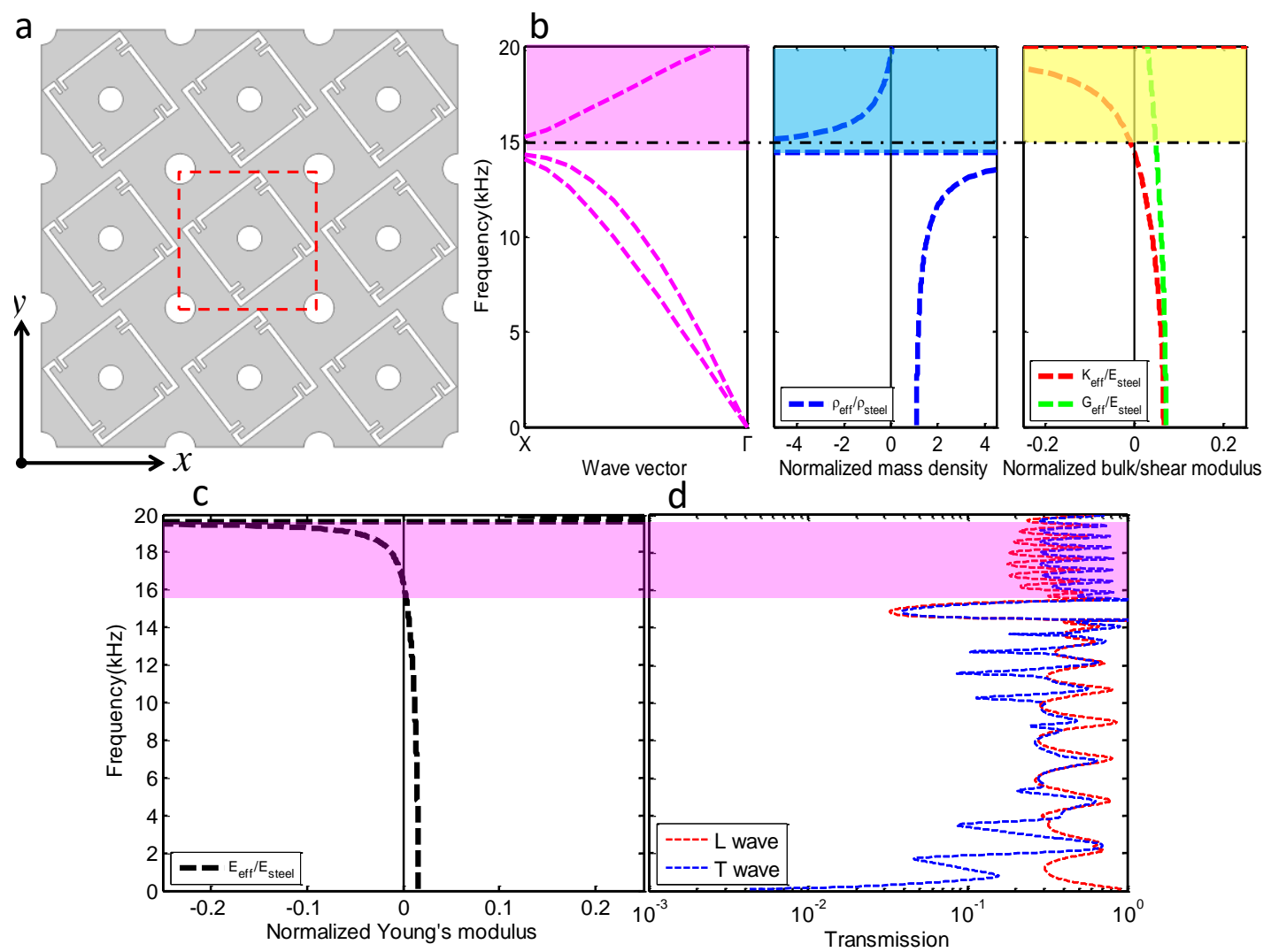

Fig. 14 Effective material parameters, the dispersion curves and the transmission property of the optimized chiral EMMs. (a) the optimized EMMs with $3 \times 3$ unit cells; (b) the double negative parameters and the dispersion curves along $\Gamma X$ direction; (c) the effective Young's modulus, and (d) the transmission property when the longitudinal wave impinges to the sample.

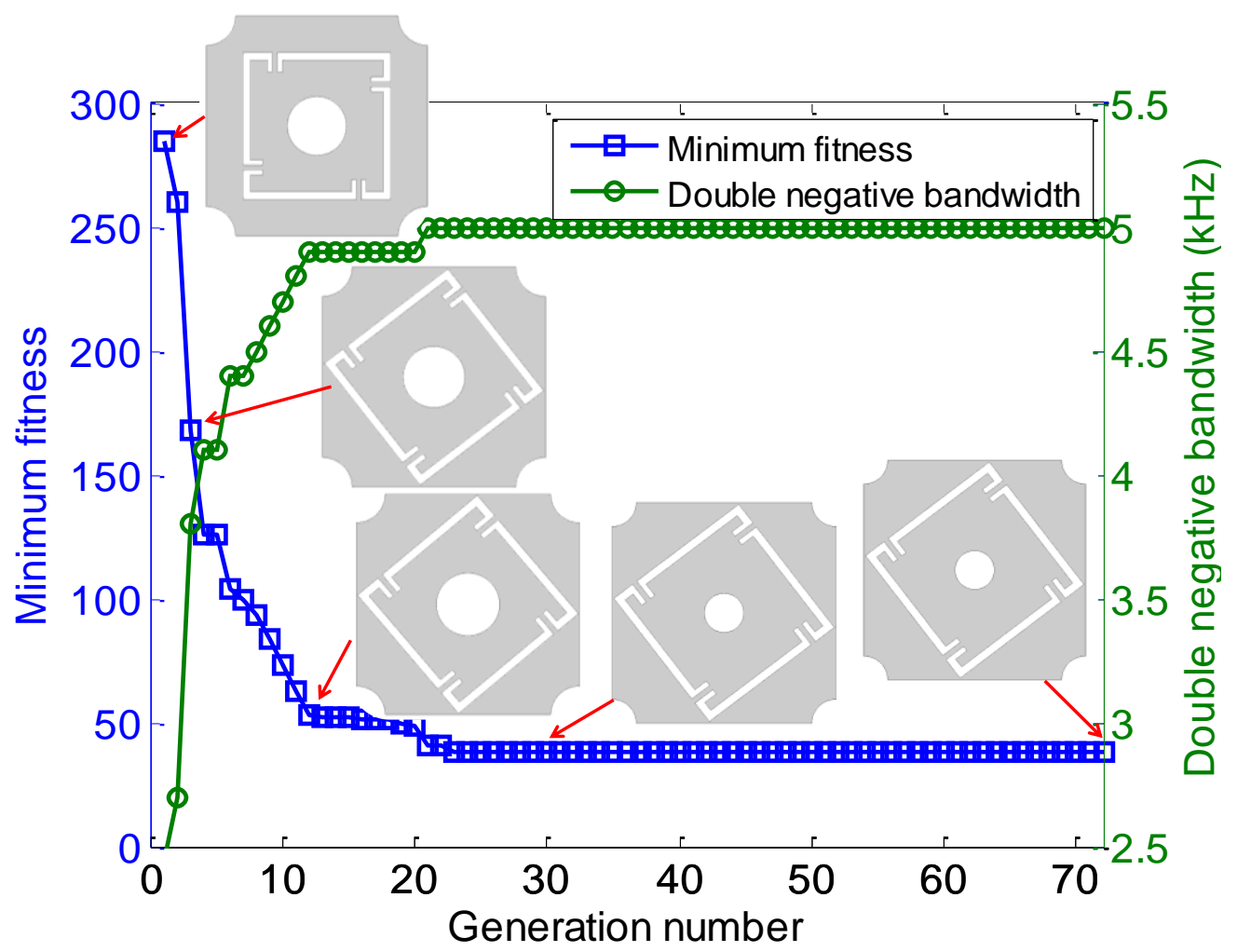

Fig. 15 The shape and the double negative bandwidth evolution with the increase of the generation 
number considering eight variables in $[0-20 \mathrm{kHz}]$.

\section{Simulations of the negative refraction and the imaging}

In this section, the prominent phenomena associated with the double negativity, which contains the negative refraction and the imaging effect, is demonstrated [46]. Numerical simulations based on the optimized structure of types (a) and (d) of the single-phase chiral material (Tab. 3) are shown in Fig. 16. The chiral EMMs sample is composed of $10 \times 64$ unit-cells with the surface normal along the $\Gamma \mathrm{X}$ direction. Then the sample is rotated 45 degrees with respect to the lower horizontal direction, and it is extended into the background medium. The sample and the background medium use the same single-phase material, which is mentioned in Tab. 1. In the harmonic numerical simulation, the Gaussian beam with an actuation length of $700 \mathrm{~mm}$ is incident along the horizontal direction. In order to suppress the reflected waves from the boundaries, the perfectly matched layers (PML) are placed around the background medium.

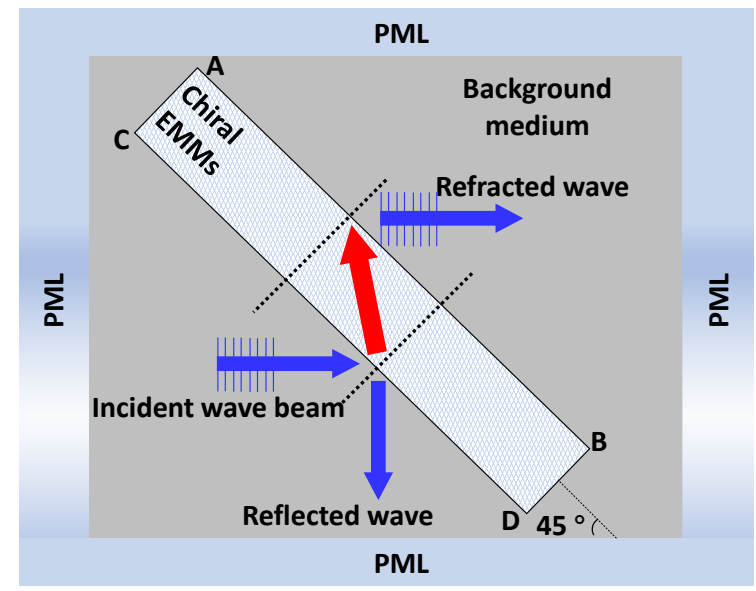

(a)

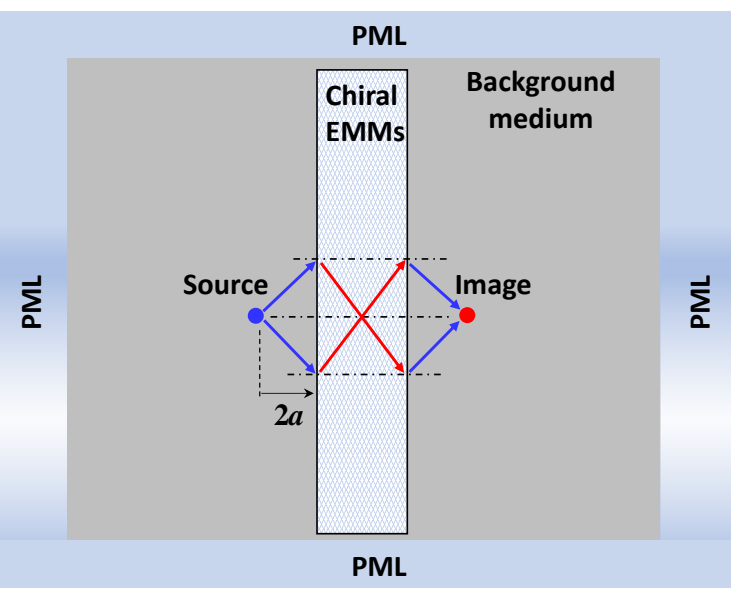

(b)

Fig. 16 Schematic of 2D numerical simulation for (a) the negative refraction and (b) the imaging effect.

Because of the vector nature of the elastic wave, the reflected/refracted longitudinal/transverse waves can be generated simultaneously at the interface of two different elastic materials [13]. Generally, the conversion efficiency is frequently used to quantify the transmission ratio of the impinging longitudinal wave ( $L$ wave) to the reflected/refracted longitudinal/transverse $(L / T)$ waves. The conversion efficiency is defined as the ratio of the energy flux distribution of the reflected/transmitted waves 
to that of the incident longitudinal wave in the far-field distributions [47]. In this case, the energy flux distribution is described by integrating the time averaged Poynting vector $\mathbf{P}$ along the outgoing direction [28], where $\mathbf{P}=\pi f \operatorname{Im}\left(\boldsymbol{\sigma} \cdot \mathbf{u}^{*}\right)$. The vectors $\mathbf{u}^{*}$ and $\boldsymbol{\sigma}$ are, respectively, the complex conjugate of the displacement vector and the stress tensor. The notation $\operatorname{Im}(\cdot)$ means the imaginary part is reserved. Then the conversion efficiency can be calculated as:

$$
C_{L-\Phi}=\bar{P}_{n}^{\alpha \Phi} / \bar{P}_{n}^{i L}
$$

where the notations $\alpha=r, t$ represent the reflected or refracted waves, and $\Phi=L, T$ denote the longitudinal or the transverse waves, respectively. $\bar{P}_{n}^{\alpha \Phi}$ is the integral of the energy flux distribution of the reflected/transmitted waves along the outgoing direction $A_{\text {out }}$, and $\bar{P}_{n}^{i L}$ denotes the integral of the energy flux distribution of the longitudinal wave along the impinging direction $A_{i n}$. They are calculated by: $\bar{P}_{n}^{\alpha \Phi}=\int_{A_{\text {out }}} \mathbf{P}^{\alpha \Phi} \cdot \mathbf{n} \mathrm{d} A, \bar{P}_{n}^{i L}=\int_{A_{\text {in }}} \mathbf{P}^{i L} \cdot \mathbf{n} \mathrm{d} A$, where $\mathbf{n}$ is the normal direction of the propagating wave, and the length of the integral is the same as the actuation length. More details regarding the computation of the conversion efficiency can be referred to $[13,47]$.

Firstly, the negative refraction of the first type of optimized EMMs at frequencies $17.5 \mathrm{kHz}, 18.0 \mathrm{kHz}, 18.5 \mathrm{kHz}$, and $19.0 \mathrm{kHz}$ are shown in Fig. 17. Note that these frequencies locate in the frequency range of the double negativity. This contour is plotted based on the displacement field of longitudinal wave component $(\nabla \cdot \mathbf{u})$. As illustrated in Fig. 17, the negative refraction is clearly observed at the interface between the metamaterial sample and the background medium. The incident wave beam is found to shift twice and horizontally propagates through the EMM sample according to the Snell's law. As the microstructure sample is oblique, the angles of incidence are all considered as 45 degrees. Then the angles of refraction are computed from the velocity field in the $2 \mathrm{D}$ wave number domain. The computations for the 
angles of refraction are partly based on [28]. It should be noted that the reflected longitudinal wave is also observed at the first interface due to the impedance mismatch. The reflected angle can be approximately evaluated as about 45 degrees.

In addition, the displacement field of the transverse wave component $(\nabla \times \mathbf{u})$ at $17.5 \mathrm{kHz}, 18.0 \mathrm{kHz}, 18.5 \mathrm{kHz}$, and $19.0 \mathrm{kHz}$ are displayed in Fig. 18. It can be found that the transverse waves are refracted into the upper right area for all the considered frequencies. From Fig. 18, the reflected transverse waves are also observed at the bottom area of the sample. The angles of refraction/reflectance of the transverse waves, which are computed based on the similar way as the computation of the refracted angles of the longitudinal wave, are denoted in the figure. The appearance of the refracted/reflected transverse waves indicates that the mode conversion from the $L$ wave to the $T$ wave has occurred at the interface.

To further quantify the conversion efficiency, Eq. (19) is used. For the considered frequencies, the values of the conversion efficiency from the incident $L$ wave to the reflected/refracted $L / T$ waves are computed, which can be seen in Tab. 5. From the table, a mini conclusion can be made, that is, most $(\geq 72 \%)$ of the energy of the impinging $L$ wave are transmitted to the energy of the $L$ wave pattern, while a small percentage $(\leq 20 \%)$ of the energy are converted to the energy of the $T$ wave pattern. It should be noted here that the sum of the conversion efficiency for a working frequency is not always $100 \%$ since part of the input energy transfers to the reflected waves inside the chiral EMMs [28].

Tab. 5 The conversion efficiency of the incident longitudinal wave to the refracted/reflected waves at the interface. $L / T$ denotes the longitudinal/transverse waves, $r$ denotes the reflected wave, and $t$ denotes the refracted wave, respectively.

\begin{tabular}{ccccc}
\hline Frequencies & $i L$ to $r L$ & $i L$ to $r T$ & $i L$ to $t L$ & $i L$ to $t T$ \\
\hline $17.5 \mathrm{kHz}$ & $43.3 \%$ & $2.4 \%$ & $28.6 \%$ & $11.7 \%$ \\
$18.0 \mathrm{kHz}$ & $33.2 \%$ & $3.5 \%$ & $39.5 \%$ & $15.3 \%$ \\
$18.5 \mathrm{kHz}$ & $21.2 \%$ & $3.8 \%$ & $49.1 \%$ & $12.3 \%$ \\
$19.0 \mathrm{kHz}$ & $29.3 \%$ & $5.6 \%$ & $49.9 \%$ & $11.6 \%$ \\
\hline
\end{tabular}

Furthermore, the imaging effect of the single-phase EMMs is investigated. Fig. 16(b) presents the schematic of $2 \mathrm{D}$ simulation for the imaging of the longitudinal wave. The source is placed at the position which is $2 a$ away from the left side of the 
sample. Here, the samples of the first and fourth types of EMMs consisting of $7 \times 50$ and $8 \times 52$ unit-cells, respectively, are applied. Besides, in order to obtain a symmetry characteristics of the propagating longitudinal wave in the EMMs along the $y$ direction, the unit-cells are set as levorotary and dextrorotary respectively in the upper and lower sides (the two sides are bisected by the white dotted line in Figs. 19(a) and 19(c)). In this case, the additional material parameters (e.g., chirality index) of the
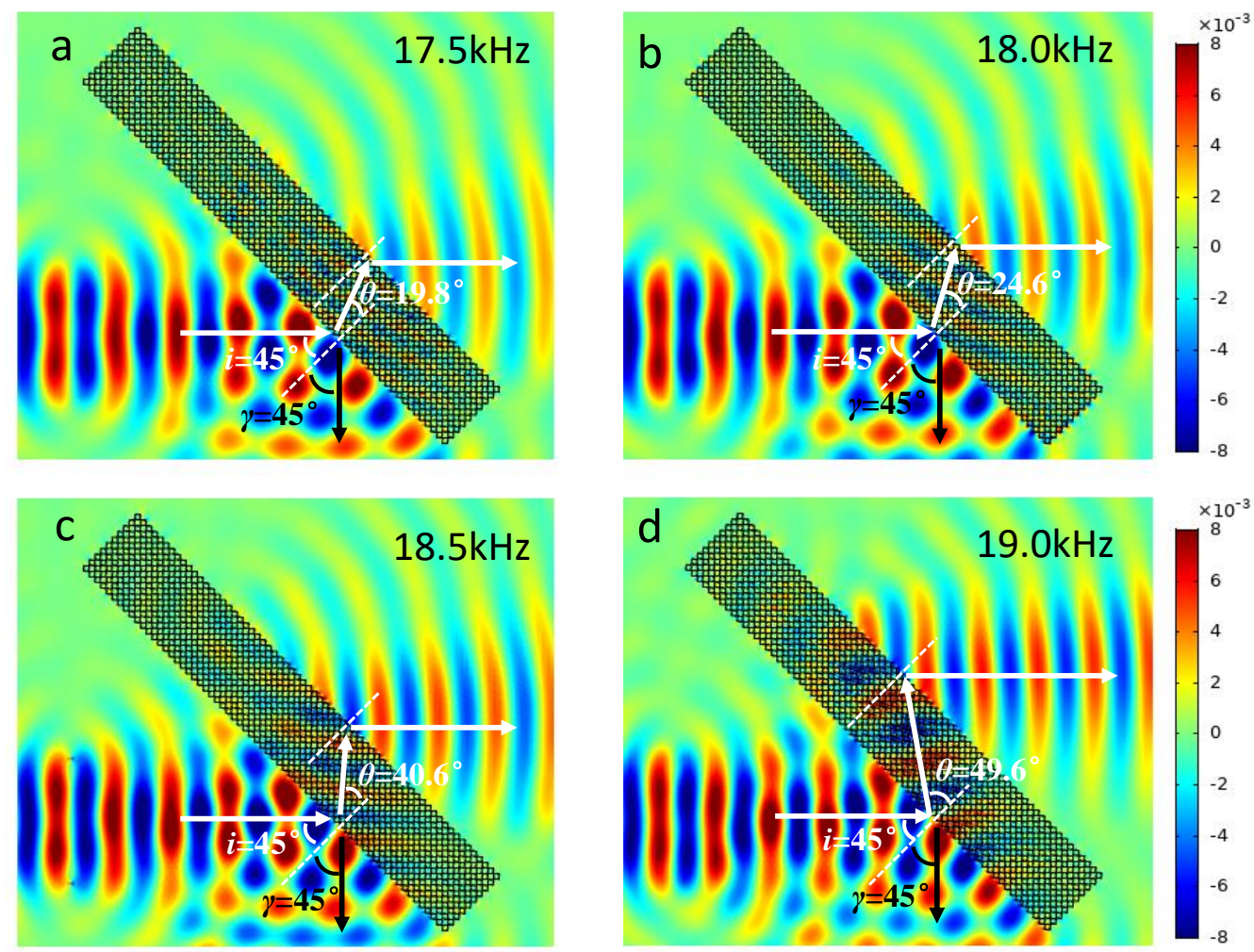

Fig. 17 Simulation of negative refraction of the first type of optimized EMMs at different frequencies. The displacement field of the longitudinal wave component ( $\nabla \cdot \mathbf{u}$ ) at: (a) $17.5 \mathrm{kHz}$, (b) $18.0 \mathrm{kHz}$, (c) $18.5 \mathrm{kHz}$, and (d) $19.0 \mathrm{kHz}$.

ortho-chiral EMMs only reverse its sign and maintain the invariance of the strain energy density, showing a $Z_{4}$-invariance in the plane as the handedness of the material pattern is flipped over [24, 26]. It can be seen from Fig. 19 that the imaging effects for the first and forth types of EMMs are clearly observed at the right side of the metamaterial sample. To confirm the subwavelength imaging effect of the chiral EMMs, the normalized intensity profiles of the image spot is expressed as a function of the normalized position. The intensity profiles are computed by using the absolute amplitude of longitudinal wave component. As shown in Figs. 19(b) and 18(d), the full width at the half maximum (FWHM) are $0.38 \lambda$ and $0.28 \lambda$ for the considered 
microstructures, where $\lambda$ represents the wavelength of the longitudinal wave propagating in the background medium. Since the FWHM are less than $0.5 \lambda$, the Rayleigh diffraction limit of elastic wave imaging is broken through. This has demonstrated that the metamaterial made by the optimized EMMs is able to achieve a super-resolution at the sub-wavelength scale.
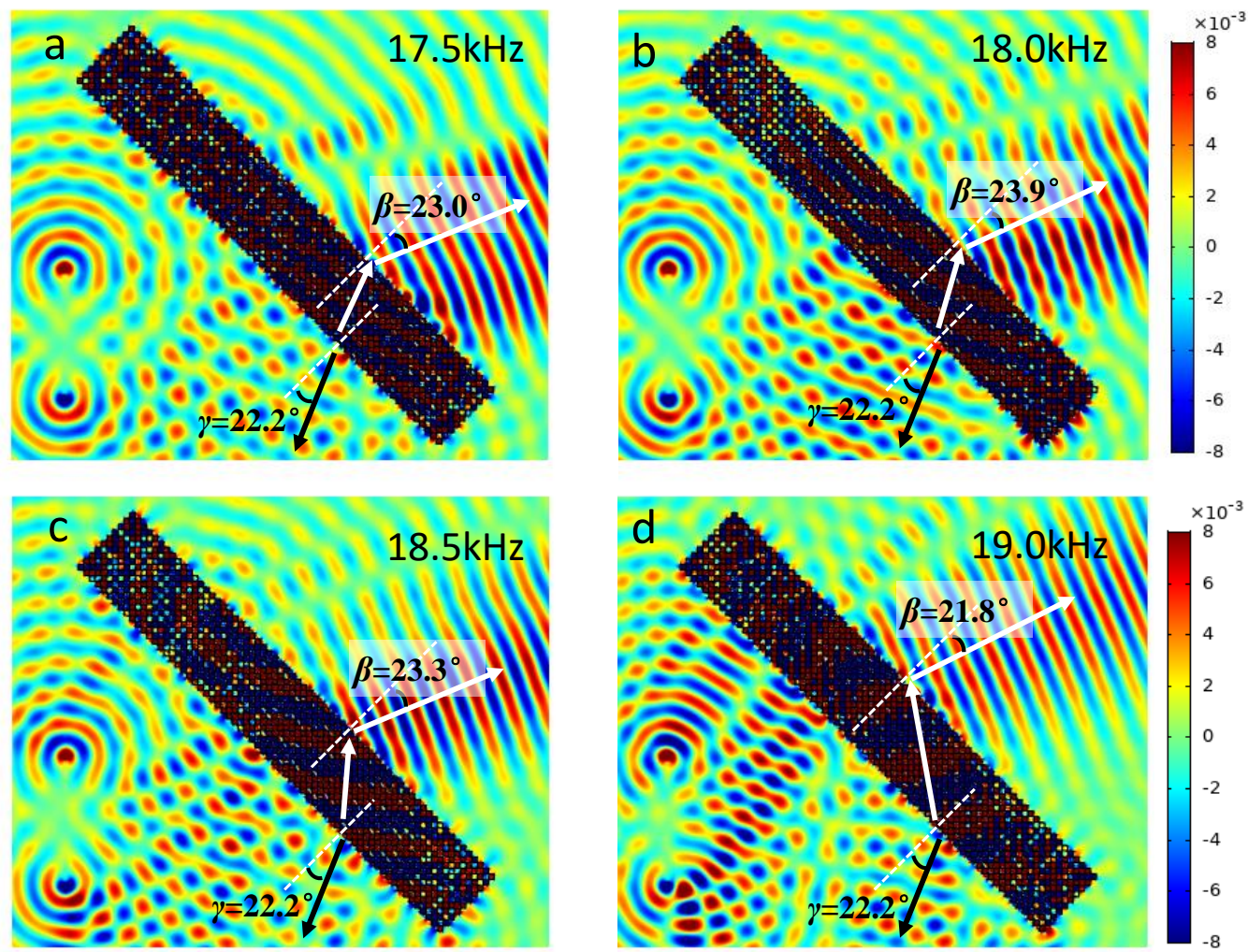

Fig. 18 Simulation of negative refraction of the first type of optimized EMMs at different frequencies.

The displacement field of the transverse wave component ( $\nabla \times \mathbf{u}$ ) at: (a) $17.5 \mathrm{kHz}$, (b) $18.0 \mathrm{kHz}$, (c) $18.5 \mathrm{kHz}$, and (d) $19.0 \mathrm{kHz}$.

In addition to the simulations based on the abovementioned optimized structures (i.e., the types (a) and (d)), the simulations of the negative refraction and the imaging of the other optimized structures (the types (b), (c) and the structures in numerical cases 2-3) are also implemented. As the process is very similar, the result comparison is made in this part. Among all the optimized structures, the optimized structures of type (d) is recommended to conduct an experiment validation in the future, as it can provide the best subwavelength-resolution imaging. The optimized structures of type (a) and that of case 3 are recommended to the realization of the negative refraction since they can afford a larger double negative frequency band. 

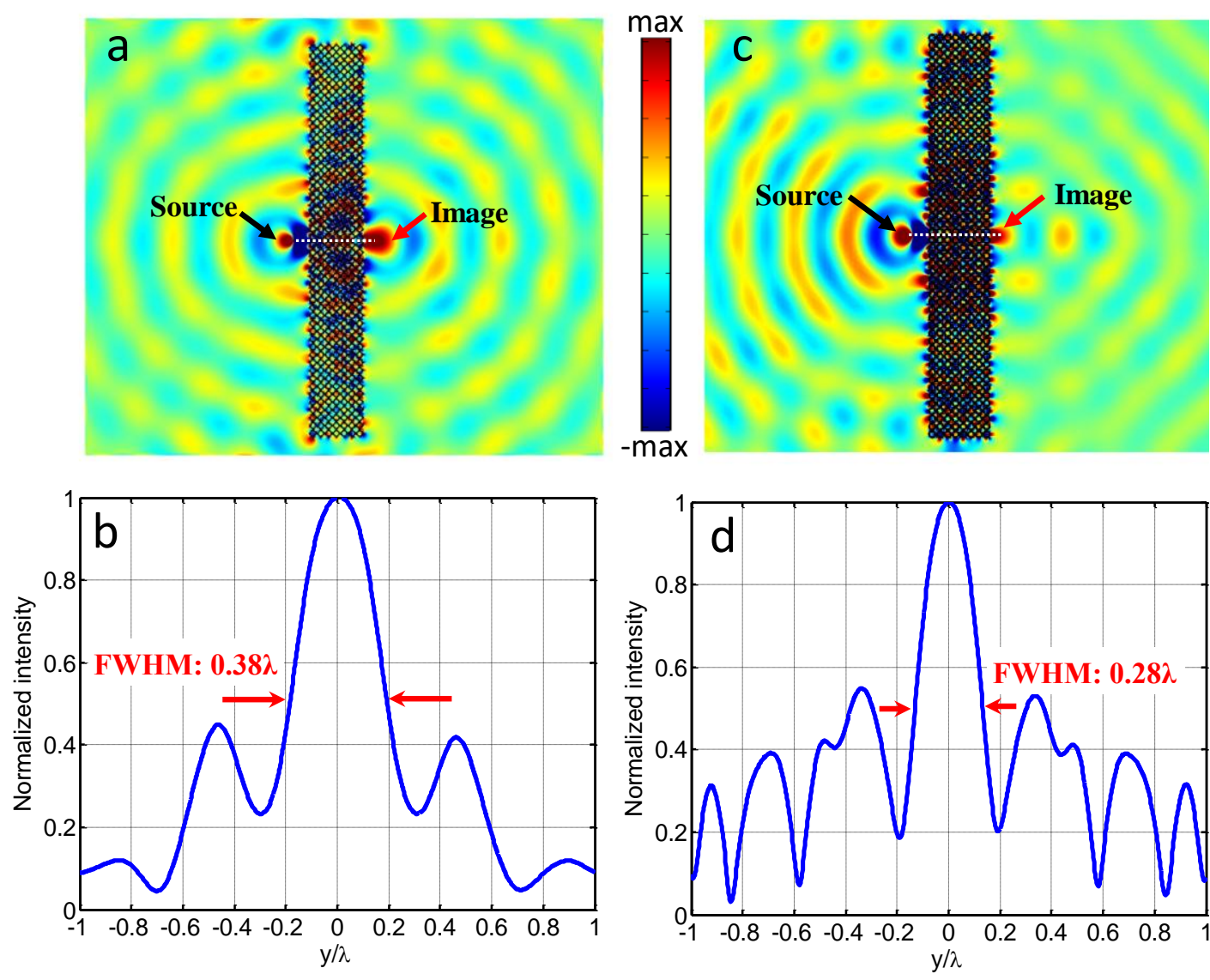

Fig. 19 Simulation of imaging effect based on the optimized single-phase chiral EMMs. (a)-(b) The imaging field pattern and the intensity profile of the image spot based on the first type of optimized EMMs at $17.85 \mathrm{kHz}$; (c)-(d) the imaging field pattern and the intensity profile of the image spot based on the fourth type of optimized EMMs at $18.52 \mathrm{kHz}$.

\section{Conclusions}

In this paper, a shape optimization strategy is presented to expand the frequency band of the double negativity based on the single-phase chiral elastic metamaterials (EMMs). Numerical cases and the simulations related to double negativity are conducted to demonstrate the proposed method and the novel metamaterial. The conclusions can be summarized as follows:

Firstly, the global sensitivity analysis reveals that the width of the rib is the most sensitive factor that influences the width of the frequency range, and the rotation angle is the least sensitive parameter. Secondly, the shape optimization with different initial shapes shows that the shape of square core with a square frame can generate the largest frequency range of double negative parameters among the considered four types of EMMs. Thirdly, the shape optimization with different target frequency ranges demonstrates that the proposed optimization strategy is able to enlarge the frequency 
band with double negativity over a lower relative frequency range. Fourthly, the numerical case with eight variables shows that the proposed method can obtain a broadband double negativity successfully when multiple design variables are considered. Finally, the applications of double negativity including the negative refraction and the imaging at a sub-wavelength scale are clearly observed. Two interesting facts are that the mode conversion has been observed at the interface, and the image resolution of the optimized chiral EMMs can reach $0.28 \lambda$, which indicates a super-resolution imaging phenomenon.

As a final remark, it is hoped that the proposed metamaterial combined with the design approach may open a novel way to manipulate the wave propagation in acoustic or elastic metamaterials.

\section{Acknowledgments}

The project is supported by the Foundation for Innovative Research Groups of the National Natural Science Foundation of China (Grant No. 51621004) and the Natural Science Foundation of China (Grant No. U1864207), the Opening Project of the Guangxi Key Laboratory of Automobile Components and Vehicle Technology of Guangxi University of Science and Technology (No. 2017GKLACVTKF01), Guangxi Science and Technology Project (No. 2017AA10104), and the opening project of the Hunan Provincial Key Laboratory of Vehicle Power and Transmission System (No. VPTS201903). We would like to thank Dr. Hao-Wen Dong (Hong Kong Polytechnic University) for his helpful discussions about the simulation of the double negativity.

\section{Conflict of interest}

On behalf of the authors, the corresponding author states that there is no conflict of interest.

\section{References}

[1] D.R. Smith, J.B. Pendry, M.C.K. Wiltshire, Metamaterials and Negative Refractive Index, Science, 305 (2004) 788.

[2] D.R. Smith, W.J. Padilla, D.C. Vier, S.C. Nemat-Nasser, S. Schultz, Composite Medium with Simultaneously Negative Permeability and Permittivity, Physical Review Letters, 84 (2000) 4184-4187. 
[3] V.G. Veselago, THE ELECTRODYNAMICS OF SUBSTANCES WITH SIMULTANEOUSLY NEGATIVE VALUES OF \$lepsilon\$ AND $\mu$, Soviet Physics Uspekhi, 10 (1968) 509-514.

[4] N. Fang, H. Lee, C. Sun, X. Zhang, Sub-diffraction-limited optical imaging with a silver superlens, Science, 308 (2005) 534-537.

[5] Y. Ding, Z. Liu, C. Qiu, J. Shi, Metamaterial with Simultaneously Negative Bulk Modulus and Mass Density, Physical Review Letters, 99 (2007) 093904.

[6] J. Li, C.T. Chan, Double-negative acoustic metamaterial, Physical Review E, 70 (2004) 055602.

[7] X. Wu, Y. Su, J. Shi, Perspective of additive manufacturing for metamaterials development, Smart Materials and Structures, 28 (2019) 093001.

[8] H.-W. Dong, S.-D. Zhao, Y.-S. Wang, C. Zhang, Topology optimization of anisotropic broadband double-negative elastic metamaterials, Journal of the Mechanics and Physics of Solids, 105 (2017) 54-80.

[9] L. Feng, X.-P. Liu, M.-H. Lu, Y.-B. Chen, Y.-F. Chen, Y.-W. Mao, J. Zi, Y.-Y. Zhu, S.-N. Zhu, N.-B. Ming, Acoustic Backward-Wave Negative Refractions in the Second Band of a Sonic Crystal, Physical Review Letters, 96 (2006) 014301.

[10] H.-W. Dong, S.-D. Zhao, P. Wei, L. Cheng, Y.-S. Wang, C. Zhang, Systematic design and realization of double-negative acoustic metamaterials by topology optimization, Acta Materialia, 172 (2019) 102-120.

[11] K. Wang, J. Zhou, C. Cai, D. Xu, H. Ouyang, Mathematical modeling and analysis of a meta-plate for very low-frequency band gap, Applied Mathematical Modelling, 73 (2019) 581-597.

[12] Y. Zhou, P. Wei, X. Zhou, Multi-displacement continuum modelling of the metamaterial plate with periodical arranged resonators, Applied Mathematical Modelling, 76 (2019) 655-668.

[13] Y. Wu, Y. Lai, Z.-Q. Zhang, Elastic Metamaterials with Simultaneously Negative Effective Shear Modulus and Mass Density, Physical Review Letters, 107 (2011) 105506.

[14] Y. Lai, Y. Wu, P. Sheng, Z.-Q. Zhang, Hybrid elastic solids, Nature Materials, 10 (2011) 620.

[15] G.W. Milton, J.R. Willis, On modifications of Newton's second law and linear continuum elastodynamics, Proceedings Of the Royal Society a-Mathematical Physical And Engineering Sciences, 463 (2007) 855-880.

[16] S.H. Lee, C.M. Park, Y.M. Seo, Z.G. Wang, C.K. Kim, Composite Acoustic Medium with Simultaneously Negative Density and Modulus, Physical Review Letters, 104 (2010) 054301.

[17] Y. Liu, X. Su, C.T. Sun, Broadband elastic metamaterial with single negativity by mimicking lattice systems, Journal of the Mechanics and Physics of Solids, 74 (2015) 158-174.

[18] R.E. Christiansen, O. Sigmund, Designing meta material slabs exhibiting negative refraction using topology optimization, Structural and Multidisciplinary Optimization, 54 (2016) 469-482. 
[19] Y.F. Li, F. Meng, S. Zhou, M.-H. Lu, X. Huang, Broadband All-angle Negative Refraction by Optimized Phononic Crystals, Scientific Reports, 7 (2017) 7445.

[20] Y. Liao, X. Zhou, Y. Chen, G. Huang, Adaptive metamaterials for broadband sound absorption at low frequencies, Smart Materials and Structures, 28 (2018) 025005.

[21] K. Yi, M. Ouisse, E. Sadoulet-Reboul, G. Matten, Active metamaterials with broadband controllable stiffness for tunable band gaps and non-reciprocal wave propagation, Smart Materials and Structures, 28 (2019) 065025.

[22] Y. Chen, G. Hu, G. Huang, A hybrid elastic metamaterial with negative mass density and tunable bending stiffness, Journal of the Mechanics and Physics of Solids, 105 (2017) 179-198.

[23] A. Spadoni, M. Ruzzene, Elasto-static micropolar behavior of a chiral auxetic lattice, Journal of the Mechanics and Physics of Solids, 60 (2012) 156-171.

[24] X.N. Liu, G.L. Huang, G.K. Hu, Chiral effect in plane isotropic micropolar elasticity and its application to chiral lattices, Journal of the Mechanics and Physics of Solids, 60 (2012) 1907-1921.

[25] S.K. Tomar, A. Khurana, Wave propagation in thermo-chiral elastic medium, Applied Mathematical Modelling, 37 (2013) 9409-9418.

[26] N. Auffray, R. Bouchet, Y. Brechet, Strain gradient elastic homogenization of bidimensional cellular media, International Journal Of Solids And Structures, 47 (2010) 1698-1710.

[27] X.N. Liu, G.K. Hu, C.T. Sun, G.L. Huang, Wave propagation characterization and design of two-dimensional elastic chiral metacomposite, Journal Of Sound And Vibration, 330 (2011) 2536-2553.

[28] R. Zhu, X.N. Liu, G.K. Hu, C.T. Sun, G.L. Huang, Negative refraction of elastic waves at the deep-subwavelength scale in a single-phase metamaterial, Nature Communications, 5 (2014) 8.

[29] L. Brillouin, Wave Propagation in Periodic Structures: Electric Filters and Crystal Lattices, McGraw-Hill Book Company, Inc., New York, (1953) 926.

[30] E. Li, Z.C. He, J.Y. Hu, X.Y. Long, Volumetric locking issue with uncertainty in the design of locally resonant acoustic metamaterials, Computer Methods in Applied Mechanics and Engineering, 324 (2017) 128-148.

[31] Z. Liu, X. Zhang, Y. Mao, Y.Y. Zhu, Z. Yang, C.T. Chan, P. Sheng, Locally Resonant Sonic Materials, Science, 289 (2000) 1734.

[32] R. Zhu, X.N. Liu, G.L. Huang, H.H. Huang, C.T. Sun, Microstructural design and experimental validation of elastic metamaterial plates with anisotropic mass density, Physical Review B, 86 (2012).

[33] X.N. Liu, G.K. Hu, G.L. Huang, C.T. Sun, An elastic metamaterial with simultaneously negative mass density and bulk modulus, Applied Physics Letters, 98 (2011) 251907.

[34] J.M. Podestá, C.M. Méndez, S. Toro, A.E. Huespe, Symmetry considerations for topology design in the elastic inverse homogenization problem, Journal of the Mechanics and Physics of Solids, 128 (2019) 54-78. 
[35] A.C. Eringen, Microcontinuum field theories: I. Foundations and solids, Springer Science \& Business Media2012.

[36] S. Lurie, Y. Solyaev, Revisiting bending theories of elastic gradient beams, International Journal of Engineering Science, 126 (2018) 1-21.

[37] Y.F. Wang, Y.S. Wang, L.T. Wang, Two-dimensional ternary locally resonant phononic crystals with a comblike coating, J. Phys. D-Appl. Phys., 47 (2014) 8.

[38] A. Saltelli, K. Aleksankina, W. Becker, P. Fennell, F. Ferretti, N. Holst, S. Li, Q. $\mathrm{Wu}$, Why so many published sensitivity analyses are false: A systematic review of sensitivity analysis practices, Environmental Modelling \& Software, 114 (2019) 29-39.

[39] E. Borgonovo, E. Plischke, Sensitivity analysis: A review of recent advances, European Journal of Operational Research, 248 (2016) 869-887.

[40] E. Borgonovo, A new uncertainty importance measure, Reliability Engineering \& System Safety, 92 (2007) 771-784.

[41] A. Saltelli, J. Marivoet, Non-parametric statistics in sensitivity analysis for model output: A comparison of selected techniques, Reliability Engineering \& System Safety, 28 (1990) 229-253.

[42] Bi-Directional Evolutionary Structural Optimization Method, Evolutionary Topology Optimization of Continuum Structures, (2010) 17-38.

[43] M. Arian Nik, K. Fayazbakhsh, D. Pasini, L. Lessard, A comparative study of metamodeling methods for the design optimization of variable stiffness composites, Composite Structures, 107 (2014) 494-501.

[44] D.E. Goldberg, J.H. Holland, Genetic Algorithms and Machine Learning, Machine Learning, 3 (1988) 95-99.

[45] H.-W. Dong, X.-X. Su, Y.-S. Wang, C. Zhang, Topological optimization of two-dimensional phononic crystals based on the finite element method and genetic algorithm, Structural and Multidisciplinary Optimization, 50 (2014) 593-604.

[46] R.E. Christiansen, O. Sigmund, Experimental validation of systematically designed acoustic hyperbolic meta material slab exhibiting negative refraction, Applied Physics Letters, 109 (2016) 101905.

[47] R.V. Craster, S. Guenneau, Acoustic metamaterials: Negative refraction, imaging, lensing and cloaking, Springer Science \& Business Media2012. 